TRANSACTIONS OF THE

AMERICAN MATHEMATICAL SOCIETY

Volume 363, Number 1, January 2011, Pages 203-228

S 0002-9947(2010)05125-6

Article electronically published on August 26, 2010

\title{
ABSENCE OF LINE FIELDS AND MAÑ́'S THEOREM FOR NONRECURRENT TRANSCENDENTAL FUNCTIONS
}

\author{
LASSE REMPE AND SEBASTIAN VAN STRIEN
}

\begin{abstract}
Let $f: \mathbb{C} \rightarrow \hat{\mathbb{C}}$ be a transcendental meromorphic function. Suppose that the finite part $\mathcal{P}(f) \cap \mathbb{C}$ of the postsingular set of $f$ is bounded, that $f$ has no recurrent critical points or wandering domains, and that the degree of pre-poles of $f$ is uniformly bounded. Then we show that $f$ supports no invariant line fields on its Julia set.

We prove this by generalizing two results about rational functions to the transcendental setting: a theorem of Mañé (1993) about the branching of iterated preimages of disks, and a theorem of McMullen (1994) regarding the absence of invariant line fields for "measurably transitive" functions. Both our theorems extend results previously obtained by Graczyk, Kotus and Świątek (2004).
\end{abstract}

\section{INTRODUCTION}

Let $f: \mathbb{C} \rightarrow \hat{\mathbb{C}}$ be a nonconstant, nonlinear entire or meromorphic function. The existence of invariant line fields (see Section 4) supported on the Julia set of $f$ is an important question which is related to density of hyperbolicity, one of the central problems in one-dimensional real and holomorphic dynamics. It is conjectured that flexible Lattès maps (see [M2]) are the only rational functions for which such line fields exist.

In the setting of transcendental meromorphic functions $f: \mathbb{C} \rightarrow \hat{\mathbb{C}}$, the situation is less clear. Indeed, it is known EL1 that there exist "pathological" entire transcendental functions that support invariant line fields on their Julia sets. Also, hyperbolicity - even structural stability — need not be dense in parameter spaces of entire transcendental functions if the set $S(f) \subset \hat{\mathbb{C}}$ of singular values of $f$ (that is, the closure of the set $\operatorname{sing}\left(f^{-1}\right)$ of critical and asymptotic values) is too large. (We explicitly note that we include $\infty$ in $S(f)$ if it is a critical or asymptotic value. Often $S(f)$ denotes only the finite singular values of $f$, but for the purposes of this article our convention is more convenient.) It is not even clear how "hyperbolicity" should be defined when $S(f) \cap \mathbb{C}$ is unbounded.

However, it is expected that such phenomena can be controlled when the functions in question are sufficiently tame, either function-theoretically or dynamically. For example, we believe that hyperbolicity is dense in the parameter spaces $M_{f}$ of entire functions with finitely many singular values that were defined in EL3.

Received by the editors October 8, 2008.

2010 Mathematics Subject Classification. Primary 37F10; Secondary 30D05, 37D25, 37F15, $37 \mathrm{~F} 35$.

This research was supported by EPSRC grant EP/E017886/1.

(C)2010 American Mathematical Society 
In this article, we will, on the other hand, prove the absence of invariant line fields for meromorphic functions under some strong dynamical conditions, but without making many function-theoretic assumptions. Let us say that $f$ is nonrecurrent if the finite part $\mathcal{P}(f) \cap \mathbb{C}$ of the postsingular set

$$
\mathcal{P}(f):=\overline{\left\{f^{n}(s): s \in S(f), n \geq 0\right\}} \subset \hat{\mathbb{C}}
$$

is bounded, and furthermore every critical point $c$ of $f$ is nonrecurrent; that is, $c \notin \overline{\left\{f^{n}(c): n>0\right\}}$.

1.1. Theorem (Absence of line fields for nonrecurrent maps). Let $f: \mathbb{C} \rightarrow \hat{\mathbb{C}}$ be a nonlinear and nonconstant meromorphic function. Suppose that $f$ is nonrecurrent and has no wandering domains, and that there is a bound on the order of all prepoles of $f$. Then the Julia set of $f$ supports no invariant line fields.

Remark. If $f$ as in the statement of the theorem had a wandering domain, then the set of limit points of the iterates on this domain would have to be bounded. It is an open question whether an entire or meromorphic function can have a wandering domain with this property (see [BH, Problem 2.87] and [B1, Question 8]).

We also remark that the assumption on wandering domains in Theorem $1.1 \mathrm{can}$ be weakened somewhat. E.g. it is enough to assume that the grand orbit of every wandering domain contains at most one singular orbit (see Theorem 7.4 for details).

There are two main ingredients in the proof of this theorem. The first is a generalization of Mañé's theorem $\mathrm{Ma}$ ] regarding pullbacks of disks that are disjoint from the $\omega$-limit sets of recurrent critical values. In particular, we prove the following.

1.2. Theorem (Hyperbolic sets). Let $f: \mathbb{C} \rightarrow \hat{\mathbb{C}}$ be a nonlinear and nonconstant meromorphic function, and suppose that $\mathcal{P}(f) \cap \mathbb{C}$ is bounded.

Furthermore, let $K \subset \mathbb{C}$ be a compact subset of the Julia set $J(f)$ with the following properties:

(a) $K$ is forward-invariant, i.e. $f(K) \subset K$;

(b) $K$ contains neither critical points nor parabolic periodic points;

(c) $K$ does not intersect the $\omega$-limit set of any recurrent critical points, or of any singular values contained in wandering domains.

Then $K$ is a hyperbolic set.

Remark 1. Recall that a hyperbolic set is a compact, forward-invariant set $K \subset \mathbb{C}$ such that, for some $\eta>1$ and some $k \in \mathbb{N}$, we have $\left|\left(f^{k}\right)^{\prime}(z)\right|>\eta$ for all $z \in K$.

Remark 2. It is clearly necessary to make some restrictions about unbounded singular orbits; consider e.g. the case of an entire function that has a nonlinearizable irrationally indifferent fixed point but no critical points. However, the assumption that $\mathcal{P}(f) \cap \mathbb{C}$ is bounded is stronger than what we need; we use it merely for convenience of statement. Compare Theorem 2.7.

The second main result concerns the absence of invariant line fields for a large class of "dynamically tame" transcendental meromorphic functions. This theorem generalizes a result of McMullen [McM2, Theorem 3.17]. It will be used in a subsequent paper $\underline{\mathrm{RvS}}$ to establish density of hyperbolicity in parameter spaces of certain real entire transcendental functions with finitely many singular values. (This is the original motivation behind our study.) 
To state the result, let us say that $f$ is measurably transitive if the radial Julia set $J_{r}(f)$ (see Section 3) has positive measure. Roughly speaking, this means that it is possible to pass from small scales to large scales by a univalent iterate near any point in $\mathbb{C}$, which is a natural hypothesis in our setting. If $f$ is measurably transitive, then $J(f)=\mathbb{C}$ and $f$ is ergodic on the Julia set; see Theorem 3.3 below.

We note that all functions with $\limsup \operatorname{dist}^{\#}\left(f^{n}(z), \mathcal{P}(f)\right)>0$ for a positive measure set of $z \in J(f)$ (where dist ${ }^{\#}$ denotes spherical distance) are measurably transitive.

1.3. Theorem (Absence of line fields). Let $f: \mathbb{C} \rightarrow \hat{\mathbb{C}}$ be a nonconstant, nonlinear meromorphic function.

Suppose that $f$ is measurably transitive, and that $f$ is not a Lattès map. Then $f$ supports no invariant line field on its Julia set.

Using Theorem 1.2 and a result of Bock Bo (see Theorem 3.3), it follows that a function satisfying the hypotheses of Theorem 1.1 either is measurably transitive, or almost every orbit accumulates at $\infty$. Hence Theorem 1.1 will be proved in Section 7 by showing that the latter set (and, in particular, the set of escaping points, which converge to infinity under iteration) also does not support invariant line fields.

Graczyk, Kotus and Świątek GKŚ] proved Theorem 1.2 under the additional assumptions that $\mathcal{P}(f)$ contains no critical points and that $J(f)=\mathbb{C}$. They also proved Theorem 1.1 for such functions, assuming that $\infty \notin \mathcal{P}(f)$ as well as an additional technical condition.

Our proof of Theorem 1.2 follows quite closely the account of Mañé's theorem given by Tan Lei and Shishikura $[\mathrm{ST}$, although additional care is required to deal with the transcendental case. Likewise, the proof of Theorem 1.3 follows McMullen's general strategy, but also contains some other ingredients: we use Nevanlinna's theorem on completely branched values, and also need to develop an argument to deal with the singularity at infinity for a transcendental meromorphic function. Martin and Mayer [MM] have given an alternative proof of Theorem 1.3 for rational functions that can also be applied to transcendental entire functions. This argument is extended in $\mathrm{MR}$ to give an alternative proof of Theorem 1.3 that also applies to Epstein's more general class of "Ahlfors islands maps".

We should mention that there are a number of other results regarding the absence of invariant line fields for transcendental entire and meromorphic functions which go in a somewhat different direction. In particular, in [UZ3, the absence of invariant line fields is shown for exponential maps that satisfy a type of ColletEckmann condition. The article R1 establishes that an invariant line field for any meromorphic function cannot be supported on the set of points that escape to infinity through logarithmic singularities. (This result will be used in the proof of Theorem 1.1 above.)

Also, our version of Mañé's theorem has connections to Kisaka's study [Ki of semi-hyperbolicity (which requires that all pullbacks of sufficiently small disks have only a bounded amount of branching, which e.g. excludes the existence of asymptotic values); see also $[\mathrm{BM}$. Okuyama $[\mathrm{O}$, Theorem 5.1] has a certain version of Mañé's theorem for functions with finitely many critical points and asymptotic values, while Kotus and Urbański [KU1] have previously treated the case of elliptic functions. 
Structure of the article. We begin by proving our version of Mañé's theorem in Section 2. Sections 3 to 5 discuss material that is not entirely new, but for which we know of no reference that presents it as we require. More precisely, Section 3 discusses radial Julia sets for general transcendental entire or meromorphic functions, which are important in the study of measurable dynamics of such functions. We also prove two basic results regarding these sets. Section 4 reviews facts about (univalent) line fields. In Section 5, we introduce and discuss the concept of "branched exceptional" values, which may be of independent interest and does not seem to appear explicitly in the literature. The main work for Theorem 1.3 is done in Section [6] where we classify all meromorphic functions that support univalent line fields near their Julia sets. We remark that the discussion of Mañé's theorem and radial Julia sets in Sections 2 and 3, on the one hand, and of invariant line fields in Sections 4 to 6 , on the other, can be read quite independently from each other. Finally, we tie up the loose strings in Section 7 .

Basic notation. We denote the complex plane by $\mathbb{C}$ and the Riemann sphere by $\hat{\mathbb{C}}=\mathbb{C} \cup\{\infty\}$. We use a number of different notions of distance in this article: Euclidean distance is denoted dist, spherical distance is denoted dist ${ }^{\#}$, and hyperbolic distance in some open set $U \subset \mathbb{C}$ is denoted dist $_{U}$. We use the analogous notation for diameters; e.g. $\operatorname{diam}_{U}(X)$ denotes the diameter of $X$ in the hyperbolic metric of $U$. The Euclidean disk of radius $\varepsilon$ around $z$ is denoted $\mathbb{D}_{\varepsilon}(z)$, while the corresponding spherical disk is $\mathbb{D}_{\varepsilon}^{\#}(z)$.

Throughout this article $f: \mathbb{C} \rightarrow \hat{\mathbb{C}}$ will be a nonconstant, nonlinear meromorphic function, unless explicitly stated otherwise. As usual, the Julia and Fatou sets of $f$ are denoted by $J(f)$ and $F(f)$, respectively. If $z \in \mathbb{C}$ and $U \subset \hat{\mathbb{C}}$ with $f^{n}(z) \in U$, then the pullback of $U$ along the orbit of $z$ is the component $W$ of $f^{-n}(U)$ containing $z$. This pullback is called univalent if $f: W \rightarrow U$ is univalent. The $\omega$-limit set of a point $z \in \mathbb{C}$ is the accumulation set of the forward orbit of $z$ under $f$.

We frequently deal with open covers of a compact set $K \subset \mathbb{C}$. The Lebesgue covering number of such a covering is the largest number $\delta$ such that the disk of radius $\delta$ around any point of $K$ is contained in some element of the open cover in question [Ke, Theorem 26].

We use the following notation to conclude proofs and results: $\mathbf{D}$ denotes the end of a proof, $\square$ indicates a result cited without proof, and $\triangle$ completes the proof of an auxiliary step within a larger argument.

\section{MAÑÉ'S THEOREM}

In this section, we do the main work for the proof of Theorem1.2 More precisely, we establish the "point version" of this theorem (Theorem 2.5). In the rational case, this states that a sufficiently small disk around any point of the compact set $K$ can be pulled back along arbitrary backwards orbits with only a bounded amount of branching. The "compact set version" of our theorem, stated in the Introduction, is deduced from the point version in the following section, as a special case of an observation about radial Julia sets.

To motivate what follows, we recall that the proof of Mañé's theorem essentially relies on the following two principles (compare [Ma, Lemmas 1 and 2]).

(I) Suppose we are given a sequence $V_{0} \leftarrow V_{1} \leftarrow V_{2} \leftarrow \cdots \leftarrow V_{n}$ of pullbacks of a disk $V_{0}$ intersecting the Julia set but not intersecting any recurrent critical 
orbits. Suppose furthermore that the $V_{j}$ are "small" for $j<n$. Then this pullback passes through every nonrecurrent critical point at most once, and hence the degree of such a pullback is at most the product over the degrees of nonrecurrent critical points.

(II) If a larger disk $\tilde{V}_{0}$ can be pulled back along the same orbit as $V_{0}$ with a bounded amount of branching, then the pullback of $V_{0}$ will be "small".

In the rational case, both these facts are true for any reasonable meaning of the word "small", e.g. in the spherical metric, but in the transcendental case, where the domain of definition of $f$ is noncompact, things are more complicated. For example, if we interpret "small" in the Euclidean metric, then there is no reason for (II) to be true, and it can be checked that (II) fails e.g. for the functions $f(z)=1 / \cos (\sqrt{z})$. (Consider preimages of a disk $D_{r / 2}(r)$, where $r>0$ is sufficiently small.) On the other hand, with respect to the spherical metric, there are a number of reasons for (I) to fail, for example if the pullback passes through transcendental singularities, critical points of arbitrarily high period, etc.

One might think that it is not necessary to consider unbounded pullbacks if we are only interested in bounded backward orbits. However, this is not true. It is useful to keep in mind the case of an exponential map $z \mapsto \exp (z)+\kappa$ with a bounded Siegel disk $U$. In this case, $K:=\partial U$ is a compact, forward invariant set, but $K$ is not a hyperbolic set. Hence any proof of Mańé's theorem will break down in this case, precisely because it may become necessary to follow pullbacks along the (unbounded) singular orbit. In fact, our results imply that the singular value is recurrent in this setting; compare Corollary 2.10 below.

The main insight we need in order to deal with these issues effectively is due to Tan Lei and Shishikura [ST]: they interpret "small" above as having bounded diameter in the hyperbolic metric of some suitable backwards-invariant open set $\Omega$. Then (II) is immediate (see Lemma 2.2), and (I) can be dealt with (given suitable hypotheses) by only considering pullbacks that follow the postsingular set, as in the following definition.

2.1. Definition (Regular points). We say that a point $z_{0} \in \mathbb{C}$ is regular if there are $\delta>0$ and $\Delta \in \mathbb{N}$ with the following property: if $n \in \mathbb{N}$ and $U$ is a connected component of $f^{-n}\left(\mathbb{D}_{\delta}\left(z_{0}\right)\right)$ with $U \cap \mathcal{P}(f) \neq \emptyset$, then $U$ is simply connected and $f^{n}: U \rightarrow \mathbb{D}_{\delta}\left(z_{0}\right)$ is a branched covering of degree at most $\Delta$. (If we want to be more specific, we also say that $z_{0}$ is $\Delta$-regular.)

Remark. This differs from the definition of regularity in GKŚ, where it was required that the map is univalent; i.e. $\Delta=1$.

Our goal now is to prove regularity of $z_{0}$ under suitable assumptions (e.g., when $z_{0}$ belongs to a set $K$ as in the statement of Theorem [1.2). We follow the ideas of [ST] quite closely, although our presentation differs somewhat. In particular, we use the following simple lemma from $[\mathrm{ST}]$.

2.2. Lemma (Preimages under maps of bounded degree). Let $N \in \mathbb{N}$, and let $0<\eta<1$. Then there is a constant $C(N, \eta)$ with the following property. Let $z_{0} \in \mathbb{C}$ and $\delta>0$. Suppose that $U \subset \mathbb{C}$ is simply connected, and let

$$
g: U \rightarrow \mathbb{D}_{\delta}\left(z_{0}\right)
$$


be a proper map of degree at most $N$. Then every component of $g^{-1}\left(\mathbb{D}_{\eta \cdot \delta}\left(z_{0}\right)\right)$ has diameter at most $C(N, \eta)$ in the hyperbolic metric of $U$.

As $\eta \rightarrow 0$ for fixed $N$, the constant $C(N, \eta)$ also tends to 0 .

An abstract version of the theorem. To clarify the structure of the proof, we begin by stating explicitly the hypotheses on the domain $\Omega$ that are needed in the proof (essentially, these are the requirements to make (I) above work; the reader will quickly recognize $(*)$ below as a type of nonrecurrence condition). We then deduce a version of Mañé's theorem under these assumptions. Afterwards, we formulate some explicit situations in which our setup applies. For the remainder of this section, let $N_{0} \in \mathbb{N}$ be the smallest number such that $\mathbb{D}$ can be covered by $N_{0}$ disks of radius $1 / 3$ with centers in $\mathbb{D}$. Recall our standing assumption that $f: \mathbb{C} \rightarrow \hat{\mathbb{C}}$ is a meromorphic, nonconstant and nonlinear function.

2.3. Definition (Regular sets). Let $S_{0}$ be a finite set of critical values of $f$ and let $D \in \mathbb{N}$ be an integer. We set

$$
N:=D^{\# S_{0}} \quad \text { and } \quad C_{0}:=N_{0} \cdot N \cdot C(N, 2 / 3) .
$$

A nonempty open set $\Omega \subset \mathbb{C}$ with $f^{-1}(\Omega) \subset \Omega$ is called an $\left(S_{0}, D\right)$-regular set if the following condition holds.

(*) Let $V \subset \Omega$ be an arbitrary simply connected domain, and let $U$ be a component of $f^{-1}(V)$. Assume that there is some $W \subset \Omega$ with $V \subset W$ and $\operatorname{diam}_{\Omega}(W) \leq 2 C_{0}$ such that the component of $f^{-1}(W)$ containing $U$ intersects $\mathcal{P}(f)$.

Then $f: U \rightarrow V$ is a proper map of degree at most $D$ with at most one branched value. (In particular, $U$ is simply connected.) Furthermore, if there is a branched value $s$ of this map, then $s \in S_{0}$ and $f^{j}(s) \notin U$ for all $j \geq 0$.

(A set that is $\left(S_{0}, D\right)$-regular for some $S_{0}$ and $D$ is called a regular set.)

Remark. The assumptions of $(*)$ are satisfied, in particular, if $\operatorname{diam}_{\Omega}(V) \leq 2 C_{0}$ and $U \cap \mathcal{P}(f) \neq \emptyset$. The slightly weaker assumption in $\left(^{*}\right)$ is introduced to allow for pullbacks that leave the postsingular set, but in a sense remain "close" to it.

Given an $\left(S_{0}, D\right)$-regular set $\Omega$ and a simply connected domain $V \subset \Omega$, let us say that a component $U$ of $f^{-1}(V)$ is a "good" preimage component of $V$ if the assumption in (*) is satisfied. (This notion depends on $S_{0}, D$ and $\Omega$. Whenever we use the term, it will be clear from the context what these are.) We remark that, by definition, only sets $V$ with $\operatorname{diam}_{\Omega}(V) \leq 2 C_{0}$ can have good preimage components.

In this situation, principle (I) can be phrased as the following simple lemma.

2.4. Lemma (Long good pullbacks have bounded branching). Suppose that $\Omega$ is an $\left(S_{0}, D\right)$-regular set and that $V_{0} \subset \Omega$ is simply connected. Consider a pullback $V_{0} \leftarrow V_{1} \leftarrow \cdots \leftarrow V_{n}$ of $V_{0}$; i.e., $V_{j+1}$ is a component of $f^{-1}\left(V_{j}\right)$. Assume that, for each $j<n$ with $V_{j} \cap \mathcal{P}(f) \neq \emptyset, V_{j+1}$ is a good preimage component of $V_{j}$.

Then all $V_{j}$ are simply connected, and $f^{n}: V_{n} \rightarrow V_{0}$ is a proper map of degree at most $N$, where $N$ is as in (2.1).

Proof. We first prove by induction that each $V_{j+1}$ is simply connected, and that $f: V_{j+1} \rightarrow V_{j}$ is a proper map, branched of degree at most $D$ at most over a single point of $S_{0}$. Indeed, suppose that we have already shown that $V_{j}$ is simply connected. If $V_{j} \cap \mathcal{P}(f)=\emptyset$, then $f: V_{j+1} \rightarrow V_{j}$ is a conformal isomorphism, and 
$V_{j+1}$ is simply connected. Otherwise, we can apply $\left(^{*}\right)$ to see that $f: V_{j+1} \rightarrow V_{j}$ has degree at most $D$ and is branched at most over a single point of $S_{0}$. This implies that $V_{j+1}$ is simply connected.

Furthermore, let $s \in S_{0}$. It follows from the final statement in $\left(^{*}\right)$ that there is at most one $j$ such that $f: V_{j+1} \rightarrow V_{j}$ is branched over $s$. Hence it follows that $\operatorname{deg}\left(f^{n}: V_{n} \rightarrow V_{0}\right) \leq D^{\# S_{0}}=N$, as claimed.

2.5. Theorem (General form of Mañé's theorem). Suppose that $\Omega$ is an $\left(N_{0}, D\right)$ regular set and let $N$ and $C_{0}$ be as in (2.1). Then every point of $\Omega$ is $N$-regular.

More precisely, let $z_{0} \in \Omega$ and choose $\delta>0$ such that $\mathbb{D}_{2 \delta}\left(z_{0}\right) \subset \Omega$ and $\operatorname{diam}_{\Omega}\left(\mathbb{D}_{2 \delta}\left(z_{0}\right)\right) \leq C_{0}$. Set $V_{0}:=\mathbb{D}_{\delta}\left(z_{0}\right)$.

Let $n \geq 0$, and suppose that $V$ is a component of $f^{-n}\left(V_{0}\right)$ with $V \cap \mathcal{P}(f) \neq \emptyset$. Then

(a) $V$ is simply connected and $\operatorname{deg}\left(f^{n}: V \rightarrow D_{0}\right) \leq N$, and

(b) $\operatorname{diam}_{\Omega}(V) \leq C_{0}$.

Proof. The proof is by induction. Both claims are trivial for $n=0$.

Let $V_{0} \leftarrow V_{1} \leftarrow \cdots \leftarrow V_{n}=V$ be the pullback of $V_{0}$ along the orbit of $V$. By part (b) of the induction hypothesis, we have $\operatorname{diam}_{\Omega}\left(V_{k}\right) \leq C_{0}$ for all $k<n$. Also $V_{k+1} \cap \mathcal{P}(f) \neq \emptyset$. Hence each $V_{k+1}$ is a good preimage component of $V_{k}$, and (国) follows from the induction hypothesis and Lemma 2.4 .

So, let us prove (B). To do so, cover the disk $V_{0}$ by $N_{0}$ disks of radius $\delta / 3$ with centers in $V_{0}$. Let $U^{1}, \ldots, U^{\ell}$ be the collection of those preimage components of any of these disks that intersect $V$. So each $U^{j}$ is a component of $f^{-n}\left(D^{j}\right)$, where $D^{j}=\mathbb{D}_{\delta / 3}\left(z^{j}\right)$ with $z^{j} \in V_{0}$, and the $U^{j}$ cover $V$. By (回), the number $\ell$ does not exceed $N_{0} \cdot N$. We claim that each $U^{j}$ has diameter at most $C(N, 2 / 3)$ in the hyperbolic metric of $\Omega$.

To show this, let us fix $j$ for the moment, and write $U:=U^{j}$. Consider the disk $\tilde{D}:=\tilde{D}_{j}:=\mathbb{D}_{\delta / 2}\left(z^{j}\right)$. Observe that $\mathbb{D}_{\delta}\left(z^{j}\right) \subset \mathbb{D}_{2 \delta}\left(z_{0}\right)$ by construction, so we will be able to apply the induction hypothesis to pullbacks of the disk $\tilde{D}$. Let

$$
\tilde{D}=: \tilde{U}_{0} \leftarrow \tilde{U}_{1} \leftarrow \cdots \leftarrow \tilde{U}_{n}
$$

be the pullback of $\tilde{D}$ along the orbit of $U$. Note that, $V_{k} \cap \tilde{U}_{k} \neq \emptyset$ for all $k$ by construction.

If $k<n$ is such that $\tilde{U}_{k} \cap \mathcal{P}(f) \neq \emptyset$, then $\operatorname{diam}\left(\tilde{U}_{k}\right) \leq C_{0}$ by part (b) of the induction hypothesis. So the set $W:=\tilde{U}_{k} \cup V_{k}$ has diameter at most $2 C_{0}$ in $\Omega$. The component of $f^{-1}(W)$ containing $\tilde{U}_{k+1}$ also contains $V_{k+1}$, and hence intersects the postsingular set. So $\tilde{U}_{k+1}$ is a good preimage component of $\tilde{U}_{k}$, and Lemma 2.4 implies that $\tilde{U}_{n}$ is simply connected and

$$
\operatorname{deg}\left(f^{n}: \tilde{U}_{n} \rightarrow \tilde{D}\right) \leq N .
$$

By Lemma 2.2, it follows that $\operatorname{diam}_{\Omega}(U) \leq \operatorname{diam}_{\tilde{U}_{n}}(U) \leq C(N, 2 / 3)$, as claimed.

Hence

$$
\begin{aligned}
\operatorname{diam}_{\Omega}(V) & \leq \operatorname{diam}_{\Omega}\left(\bigcup_{j=1}^{\ell} \overline{U^{j}}\right) \leq \sum_{j=1}^{\ell} \operatorname{diam}_{\Omega}\left(U^{j}\right) \\
& \leq \ell \cdot C(N, 2 / 3) \leq N \cdot N_{0} \cdot C(N, 2 / 3)=C_{0} .
\end{aligned}
$$

The proof of the induction step is complete. 
An explicit version of Mañé's theorem. We will now discuss under which hypotheses it is possible to construct a regular set $\Omega$. To do so, we introduce the following definitions.

2.6. Definition (Recurrence). Let $s \in S(f) \cap \mathbb{C}$, and let $V$ be a simply connected neighborhood of $s$. We say that a component $U$ of $f^{-1}(V)$ is unbranched if $f: U \rightarrow$ $V$ is univalent. Otherwise we say that $U$ is singular. More generally, we say that a component $U$ of $f^{-1}(V)$ is $d$-controlled if $f: U \rightarrow V$ is proper of degree at most $d$ and has no branched points except possibly over $s$.

The singular value $s$ is nonrecurrent if there is some $\delta>0$ such that no singular preimage of $V=\mathbb{D}_{\delta}(s)$ intersects the forward orbit of $s$. (Otherwise $s$ is called recurrent.)

We also say that $s$ is strongly nonrecurrent if furthermore there is $d_{s} \in \mathbb{N}$ such that every component of $f^{-1}(V)$ that intersects $\mathcal{P}(f)$ is $d_{s}$-controlled. (Otherwise, we call $s$ almost recurrent.)

Remark. If $\mathcal{P}(f) \cap \mathbb{C}$ is bounded, then there are at most finitely many recurrent singular values, and every nonrecurrent singular value is strongly nonrecurrent.

For $z_{0} \in J(f) \cap \mathbb{C}$ and $\delta>0$, let us define

$$
S_{\delta}\left(z_{0}\right):=\overline{\left\{s \in S(f): \exists j \geq 0:\left|f^{j}(s)-z_{0}\right| \leq \delta\right\}} .
$$

Let us also say that a wandering domain $G$ is "bad" if $f$ is not a covering map from $G$ to the Fatou component containing $f(G)$ and furthermore $G \cap \mathcal{P}(f) \neq \emptyset$. Note that the grand orbit of a bad wandering domain must contain at least two different singular values.

2.7. Theorem (Explicit hypotheses for Mañé's Theorem). Let $z_{0} \in J(f) \cap \mathbb{C}$ such that $z_{0}$ is neither a parabolic periodic point nor a limit point of the forward orbit of a bad wandering domain.

Suppose furthermore that for some $\delta>0$, the set $S_{1}:=S_{\delta}\left(z_{0}\right)$ is bounded, and that every $s \in S_{1}$ is strongly nonrecurrent.

Then there exist a finite subset $S_{0} \subset S_{1}$, a number $D \in \mathbb{N}$ and an $\left(S_{0}, D\right)$-regular set $\Omega \ni z_{0}$. In particular, $z_{0}$ is regular by Theorem 2.5 .

Remark. It is not strictly necessary to assume that $S_{1}$ is bounded; we could allow unbounded singular sets if we were willing to introduce additional technical assumptions.

Likewise, we could still relax the definition of bad wandering domains somewhat, e.g. allowing components that intersect the postsingular set to be mapped as a branched covering with at most one branched point and bounded degree.

However, it seems to us that the extra generality obtained would not justify the additional technicality of assumptions.

Proof. Let $s \in S_{1}$. Then there are $\delta_{s}>0$ and $d_{s} \in \mathbb{N}$ such that every singular preimage component of $\mathbb{D}_{\delta_{s}}(s)$ that intersects $\mathcal{P}(f)$ is $d_{s}$-controlled and disjoint from the forward orbit of $s$.

Since $S_{1}$ is compact, we can pick a finite set $S_{0} \subset S_{1}$ such that the collection of disks $\mathbb{D}_{\delta_{s}}(s)$ with $s \in S_{0}$ covers $S_{1}$. Let $\delta$ be the Lebesgue covering number of this covering.

Now let $D:=\max _{s \in S_{0}} d_{s}$, and set $N:=D^{\# S_{0}}$ and $C_{0}:=N_{0} \cdot N \cdot C(N, 2 / 3)$, as in (2.1). 
To construct the domain $\Omega$, let $\mathcal{O}$ be a repelling periodic orbit of period at least 3 that is not contained in the orbit of $z_{0}$. Then the backward orbit of $\mathcal{O}$ is dense in the Julia set by the Picard and Montel theorems (compare Section 5).

Consequently, if we choose $\varepsilon>0$ small enough and $M>0$ sufficiently large, then the domain $\Omega_{1}:=\mathbb{C} \backslash f^{-M}(\mathcal{O})$ will have the following properties. If $s \in S_{1}$ with $\operatorname{dist}(s, J(f))<\varepsilon$, then

$$
\left\{z: \operatorname{dist}_{\Omega_{1}}(z, s) \leq 2 C_{0}\right\} \subset \mathbb{D}_{\delta}(s) ;
$$

while for $s \in S_{1}$ with $\operatorname{dist}(s, J(f)) \geq \varepsilon$,

$$
\left\{z: \operatorname{dist}_{\Omega_{1}}(z, s) \leq 2 C_{0}\right\} \subset F(f) .
$$

(This follows readily from standard estimates on the hyperbolic metric; compare e.g. [McM2, Section 2.2].)

Now consider the subset $S_{2} \subset S_{1}$ consisting of all singular values $s \in S_{1}$ with $\operatorname{dist}(s, J(f)) \geq \varepsilon$ that are not contained in a good (i.e., not bad) wandering domain. Then $S_{2}$ is a compact subset of the Fatou set and hence is contained in finitely many Fatou components $U_{1}, \ldots, U_{n}$. Each of these Fatou components is one of the following.

- An attracting domain, a Siegel disk or a Herman ring, or an iterated preimage of such a domain. In each case, the orbit of $S_{2} \cap U_{j}$ is compactly contained in the Fatou set.

- A parabolic domain, an iterated preimage of such a domain, or a bad wandering domain. In this case, the forward orbit of $S_{2} \cap U_{j}$ may accumulate at a parabolic point, at infinity or at some other points of the Julia set. However, it does not accumulate on $z_{0}$ by assumption.

- A Baker domain, or an iterated preimage of such a domain. I.e., $f$ is a transcendental meromorphic function, $U_{j}$ is a (pre)periodic Fatou component and $\infty$ is a limit function of the sequence $\left.f^{n}\right|_{U_{j}}$. We claim that the forward orbit of $S_{2} \cap U_{j}$ cannot accumulate at $z_{0}$ in this case either.

To show this, let $V_{0} \rightarrow V_{1} \rightarrow \cdots \rightarrow V_{p}=V_{0}$ be the periodic orbit of Fatou components to which $U_{j}$ eventually maps, and let $a_{i} \in \hat{\mathbb{C}}$ be the limit function of the sequence $\left.f^{p n}\right|_{V_{i}}$. Then $a_{i}=\infty$ for at least one $i$. Furthermore, if $a_{i} \in \mathbb{C}$, then $a_{i+1}=f\left(a_{i}\right)$, and if $a_{i}=\infty$, then $a_{i+1}$ is an asymptotic value of $f$; more precisely, there is a curve to infinity in $V_{i}$ whose image is a curve in $V_{i+1}$ ending at $a_{i+1}$. (See [B1, Theorem 13].)

Since the cycle of Baker domains contains points of the postsingular set by assumption, such an asymptotic value $a_{i+1}$ cannot be strongly nonrecurrent. Indeed, if we take a preimage of a disk around $a_{i+1}$ that contains a tail of the aforementioned curve, then this preimage component is not mapped as a branched covering, but contains postsingular points.

To summarize, every finite limit point of the sequence $\left.f^{n}\right|_{U_{j}}$ lies in the forward orbit of some asymptotic value that is recurrent or almost recurrent. By assumption, $z_{0}$ cannot be one of these limit points.

It follows that

$$
z_{0} \notin A:=\overline{\bigcup_{j \geq 0} f^{j}\left(S_{2}\right)}
$$


We now set

$$
\Omega:=\Omega_{1} \backslash\left(A \cup \overline{\bigcup_{j \geq 0} f^{j}\left(S(f) \backslash S_{1}\right)}\right) .
$$

Then $z_{0} \in \Omega$; since $\Omega$ is the complement of a forward invariant set, we also have $f^{-1}(\Omega) \subset \Omega$. It remains to establish the condition $(*)$ from Definition 2.3 .

Suppose that $U, V, W \subset \Omega$ are as in $\left(^{*}\right)$. If $V \cap S(f)=\emptyset$, then (since $V$ is simply connected) $f: U \rightarrow V$ is a conformal isomorphism. Otherwise, $V$ contains some singular value $s$; by construction we must have $s \in S_{1} \backslash S_{2}$.

If $\operatorname{dist}(s, J(f))<\varepsilon$, then (2.2) and the fact that $\operatorname{diam}_{\Omega}(W) \leq 2 C_{0}$ imply that $W \subset \mathbb{D}_{\delta}(s) \subset \mathbb{D}_{\delta_{s_{0}}}\left(s_{0}\right)$ for some $s_{0} \in S_{0}$. By assumption, the component of $f^{-1}\left(\mathbb{D}_{\delta_{s_{0}}}\left(s_{0}\right)\right)$ containing $U$ intersects the postsingular set. If this component is unbranched, then $f: U \rightarrow V$ is univalent. Otherwise, by construction the map $f: U \rightarrow V$ is branched only over $s_{0}$, and of degree at most $D$, as claimed.

On the other hand, if $\operatorname{dist}(s, J(f)) \geq \varepsilon$, then by definition of $S_{2}$, the singular value $s$ is contained in a wandering domain $G$, and this domain is not bad. By (2.3), we then have $W \subset G$. So the component of $f^{-1}(G)$ containing $U$ intersects the postsingular set. By the definition of bad wandering domains, this component is mapped onto $G$ as a holomorphic covering map. Since $V$ is simply connected, it follows that $f: U \rightarrow V$ is univalent.

This completes the proof of the theorem.

The following standard lemma will be used to apply Theorem 2.7

2.8. Lemma (Bounded pullbacks). Suppose that $z_{0} \in \mathbb{C}$ is a regular point, and let $\varepsilon>0$ and $R>0$. Then there are $\delta>0$ and $D \in \mathbb{N}$ with the following property:

If $n \in \mathbb{N}$ and $V$ is a component of $f^{-n}\left(\mathbb{D}_{\delta}\left(z_{0}\right)\right)$ with

$$
f^{j}(V) \cap \mathbb{D}_{R}(0) \neq \emptyset
$$

for $j=0, \ldots, n$, then $\operatorname{diam}(V) \leq \varepsilon$ and

$$
\operatorname{deg}\left(f: V \rightarrow \mathbb{D}_{\delta}\left(z_{0}\right)\right) \leq D .
$$

Proof. Let $\Delta$ and $\delta_{0}$ be the constants from the regularity of $z_{0}$, and let $d$ be the largest degree of a critical point $c \in \overline{\mathbb{D}_{R}(0)} \backslash \mathcal{P}(f)$. We set $D:=d \cdot \Delta$.

Pick $\rho>0$ such that, for every $w \in \overline{\mathbb{D}_{R}(0)}$, every component $U$ of $f^{-1}\left(\mathbb{D}_{\rho}(w)\right)$ with $U \cap \mathbb{D}_{R}(0) \neq \emptyset$

- is simply connected,

- is mapped as a proper map by $f$,

- contains at most one critical point of $f$, and no critical points that are outside of $\overline{\mathbb{D}_{R}(0)}$.

By assumption, we know that every component of $f^{-n}\left(\mathbb{D}_{\delta_{0}}\left(z_{0}\right)\right)$ that intersects $\mathcal{P}(f)$ is simply connected and mapped by $f^{n}$ with degree at most $\Delta$. By Lemma 2.2. it follows that, for sufficiently small $\delta_{1}<\delta_{0}$, every component $U$ of $f^{-n}\left(\mathbb{D}_{\delta_{1}}\left(z_{0}\right)\right)$ that intersects $\mathcal{P}(f)$ and $\mathbb{D}_{R}(0)$ has diameter at most $\rho$.

Now let $\tilde{V}$ be a component of $f^{-n}\left(\mathbb{D}_{\delta_{1}}\left(z_{0}\right)\right)$ with $f^{j}(\tilde{V}) \cap \mathbb{D}_{R}(0) \neq \emptyset$ for $j=$ $0, \ldots, n$. Then it follows from the above that $\tilde{V}$ is simply connected and

$$
\operatorname{deg}\left(f^{n}: \tilde{V} \rightarrow \mathbb{D}_{\delta_{1}}\left(z_{0}\right)\right) \leq d \cdot \Delta=D .
$$

Using Lemma 2.2 again, we can pick a sufficiently small $\delta<\delta_{1}$ (independent of $n$ ) such that also $\operatorname{diam}(V) \leq \varepsilon$. 


\section{Applications to boundaries of Siegel disks.}

2.9. Corollary (Recurrent singular values and Siegel disks). Let $f: \mathbb{C} \rightarrow \mathbb{C}$ be a nonconstant, nonlinear meromorphic function with finitely many singular values, and let $U$ be a Siegel disk of $f$.

Then $\partial U$ is contained in the limit set of a recurrent or almost recurrent singular value of $f$.

Remark. An analogous statement holds for Herman rings, where the boundary will be contained in the limit sets of one or two recurrent or almost recurrent singular values.

Proof. Suppose that there is some $z_{0} \in \partial U$ not contained in the limit set of any recurrent or almost recurrent singular value and that $z_{0}$ is not a parabolic periodic point. Since functions with a finite set of singular values have no wandering domains BKL, we are in a position to apply Theorem 2.7. It follows that there is a small disk $D$ around $z_{0}$ such that any pullback of $D$ along the postsingular set and, in particular, along $\partial D$, undergoes at most a finite amount of branching. Using standard distortion estimates [CJY, Lemma 2.2] and the fact that $z_{0}$ is in the Julia set, we easily see that the spherical diameter of such pullbacks shrinks to zero along any backward orbit in $\partial D$, which contradicts the fact that $D$ intersects the Siegel disk $U$.

It follows that $\partial U$ is contained in the union of the limit sets of finitely many recurrent or almost recurrent singular values. That one of these contains the whole boundary follows, as in the rational case, from the fact that $f$ is ergodic with respect to harmonic measure on $\partial U$.

As far as we know, the following corollary is new even for exponential and trigonometric functions.

2.10. Corollary (Siegel disks of certain meromorphic functions). Suppose that $f$ is either an exponential map, $f(z)=\exp (z)+\kappa$, or a nonconstant, nonlinear meromorphic function with no asymptotic values and finitely many critical values. Assume also that the degrees of the critical points of $f$ are uniformly bounded by some constant $\Delta$.

Then the boundary of any Siegel disk of $f$ is contained in the limit set of some recurrent singular value.

Proof. Under the given assumptions, every nonrecurrent singular value is also strongly nonrecurrent, so we can apply the previous corollary.

We remark that it would be nice to avoid the requirement of strong nonrecurrence in Corollary 2.9. For example, consider the maps $b z \exp (z)+a$, which have one asymptotic value at $a$ and one critical value at $v=a-b / e$. Suppose that both singular values are nonrecurrent, but that $v$ accumulates on $a$. In such a situation, our theorem does not apply, but it does not seem unreasonable to expect that some form of Mañé's theorem still holds.

\section{RADial Julia SETS}

3.1. Definition (Radial Julia sets). Let $f: \mathbb{C} \rightarrow \hat{\mathbb{C}}$ be a nonconstant and nonlinear meromorphic function. The radial Julia set $J_{r}(f)$ is the set of all points $z \in J(f)$ with the following property: there is some $\delta>0$ such that, for infinitely many 
$n \in \mathbb{N}$, the spherical disk $\mathbb{D}_{\delta}^{\#}\left(f^{n}(z)\right)$ can be pulled back univalently along the orbit of $z$.

Remark 1. If $z \in J(f)$ and limsup $\operatorname{dist}^{\#}(z, \mathcal{P}(f))>0$, then $z \in J_{r}(f)$, since a disk that does not intersect $\mathcal{P}(f)$ can be pulled back univalently along any backward orbit.

Remark 2. It is essential to use the spherical metric in the above definition, rather than e.g. the Euclidean metric. For example, consider the function $f(z)=\sin (z) / 2$, for which the origin attracts both critical values of $f$. Setting $\delta:=\operatorname{dist}(\mathcal{P}(f), J(f))$, it follows that any disk of radius $\delta$ around some point of the Julia set can be pulled back univalently along any backward orbit. By a result of McMullen McM1, the Julia set $J(f)$ has positive area. However, by Theorem 3.3, the area of the radial Julia set $J_{r}(f)$, as defined above, is zero.

Measurably transitive functions. It is well known that $J_{r}(f)$ has either full or zero Lebesgue measure and that, in the former case, almost every orbit is dense in the plane. (For rational functions, this fact can be found in [EL2, page 608], while it was proved for meromorphic functions by Bock [Bo.) As mentioned in the Introduction, we will call $f$ measurably transitive if $J_{r}(f)$ has positive (and hence full) measure.

Since $[\mathrm{BO}$ is not widely available, let us sketch a proof of the above-mentioned result, following McMullen's argument [McM2, Theorem 3.9] for the rational case. We begin with a preliminary observation.

3.2. Lemma and Definition (Pullbacks shrink). Let $z \in J_{r}(f)$. Then there exists a disk $D=\mathbb{D}_{\delta}^{\#}(\zeta)$ such that, for infinitely many $n$, the point $f^{n}(z)$ belongs to $D$ and the larger disk $\mathbb{D}_{2 \delta}^{\#}(\zeta)$ pulls back univalently along the orbit of $z$.

In particular, the pullbacks $D_{n} \ni z$ of $D$ have diameter tending to zero, and $f^{n}: D_{n} \rightarrow D$ has uniformly bounded distortion.

(We will refer to $D$ as a disk of univalence for $z$.)

Proof. Let $\delta^{\prime}$ be the constant from Definition 3.1, and let $n_{k}$ be a sequence such that $\mathbb{D}_{\delta^{\prime}}\left(f^{n_{k}}(z)\right)$ can be pulled back univalently to $z$. Choosing $\zeta$ to be a limit point of the sequence $f^{n_{k}}(z)$ and letting $\delta<\delta^{\prime} / 2$ proves the first claim.

The fact that the $f^{n}$ have uniformly bounded distortion on $D_{n}$ follows from Koebe's distortion theorem [Po, Theorem 1.3]. If $\operatorname{diam}\left(D_{n}\right)$ did not tend to zero, then there would be a disk $U$ around $z$ with $f^{n}(U) \subset D$ for infinitely many $n$, which is impossible since $z \in J(f)$.

3.3. Theorem (Ergodicity). Suppose that $f$ is measurably transitive; that is, $J_{r}(f)$ has positive measure. Then $J_{r}(f)$ has full measure in $\hat{\mathbb{C}}$ (in particular, $J(f)=\mathbb{C}$ ), and almost every point $z \in \mathbb{C}$ has a dense orbit. Furthermore, any set that is forward invariant under $f$ has either full or zero Lebesgue measure; in particular, the action of $f$ on $\mathbb{C}$ is ergodic.

Proof. Notice that $J_{r}(f)$ is forward-invariant. Let $F$ be any forward-invariant set such that $X:=F \cap J_{r}(f)$ has positive measure. We will show that $X$ has full Lebesgue measure in the sphere.

Let $z$ be a Lebesgue density point of $X$, and let $D$ be a disk of univalence for $z$. Then

$$
\frac{\operatorname{meas}\left(X \cap D_{n}\right)}{\operatorname{meas}\left(D_{n}\right)} \rightarrow 1
$$


(where $D_{n}$ is the pullback from Lemma 3.2). Since the distortion of $\left.f^{n}\right|_{D_{n}}$ is bounded independently of $n$, it follows that

$$
\frac{\operatorname{meas}(D \backslash X)}{\operatorname{meas}(D)} \leq K \cdot \frac{\operatorname{meas}\left(D_{n} \backslash X\right)}{\operatorname{meas}\left(D_{n}\right)} \rightarrow 0
$$

for some constant $K$. Hence meas $(X \cap D)=\operatorname{meas}(D)$; i.e., $X$ has full measure in $D$. As $X$ is forward-invariant and $D$ is an open disk intersecting the Julia set, it follows that $X$ has full Lebesgue measure in the plane.

In particular, $J_{r}(f)$ itself has full measure, and any forward-invariant set has either full or zero measure in the plane. Note furthermore that the set of points whose orbits never enter a given open set $U \subset \hat{\mathbb{C}}$ is forward-invariant. Since this set is disjoint from the positive measure set $U$, it must have zero Lebesgue measure. Hence almost every point has a dense orbit.

Remarks about the definition of the radial Julia set. Radial Julia sets, as the sets where it is possible to go from small to large scales via univalent iterates, were originally introduced for rational functions. The concept of measurable transivity appears to have made its first (implicit) appearance in $[\mathrm{L}$, while the radial Julia set was explicitly introduced in DMNU, McM3. The name "conical Julia set" is also sometimes used (there are in fact a number of different definitions of conical Julia sets; see $\mathrm{Pr}$ for a discussion and interesting results about their relation to each other); the term "radial Julia set" was coined by McMullen McM3 who used it in analogy to his studies of Kleinian groups.

The radial Julia set plays a fundamental role in the study of measurable properties of conformal dynamical systems, particularly in the transcendental case, where $J_{r}(f)$ may frequently be much smaller than $J(f)$. E.g., $J(f)$ may have positive measure, while $J_{r}(f)$ has Hausdorff dimension strictly less than two; see below. (Work by Avila and Lyubich [AL suggests that this may also happen for rational functions, even quadratic polynomials. On the other hand, it follows from recent work of Buff and Chéritat $\mathrm{BC}$ that there are also quadratic polynomials for which $J(f)$ has positive measure and $J_{r}(f)$ has Hausdorff dimension 2.) In the rational case, it is known DMNU] that the Hausdorff dimension of $J_{r}(f)$ coincides with several other dynamically important quantities, including Shishikura's hyperbolic dimension, the supremum over the dimensions of all hyperbolic subsets of $J(f)$.

In the transcendental setting, the fact that the hyperbolic dimension need not equal the dimension of the full Julia set is implicit already in the work of Stallard [St]. (Stallard discusses critical Poincaré exponents rather than the hyperbolic dimension, but it is easy to see that the former is an upper bound for the latter.) A closer investigation of this phenomenon was initiated by Urbański and Zdunik [UZ1, who studied the case where $f$ is a hyperbolic exponential map $f(z)=\lambda \exp (z)$ with a single attracting basin. Here, $J_{r}(f)$ is $J(f)$ minus the set

$$
I(f):=\left\{z \in \mathbb{C}: f^{n}(z) \rightarrow \infty\right\}
$$

of escaping points, and Urbański and Zdunik prove a number of fundamental results. In particular, they show that the Hausdorff dimension $h$ of $J_{r}(f)$ lies strictly between 1 and 2 (while $J(f)$ has dimension 2 by a result of McMullen) and agrees with the hyperbolic dimension of $f$. They also construct natural conformal measures and invariant measures supported on the set $J_{r}(f)$. 
In UZ2, a similar program was carried out for the nonhyperbolic case of an exponential map whose singular value 0 escapes to infinity "sufficiently fast" under iteration; here $J_{r}(f)$ is the set of points that do not accumulate on the singular orbit. This program has subsequently been adapted to a variety of situations where there is good control over the postsingular set, and it seems likely that a good understanding of the set $J_{r}(f)$ will be the key to studying the measurable dynamics of more general transcendental functions. It is possible to show R2 that, as in the rational case, the hyperbolic dimension of $f$ and the Hausdorff dimension of $J_{r}(f)$ always coincide. In this context, let us note the following simple fact, which we will use to deduce Theorem 1.2 from the results of the previous section.

3.4. Lemma (Hyperbolic sets and $\left.J_{r}(f)\right)$. Let $K \subset \mathbb{C}$ be a hyperbolic set. Then $K \subset J_{r}(f)$. Conversely, every compact, forward-invariant set $K$ with $K \subset J_{r}(f)$ is a hyperbolic set.

Proof. Let $K$ be a hyperbolic set. Then we can pick $k \in \mathbb{N}$ and a bounded open neighborhood $W$ of $K$ such that $\left|\left(f^{k}\right)^{\prime}(z)\right|>\eta>1$ for all $z \in W$. For every $w \in f^{k}(K)$, pick an open neighborhood $U(w)$ small enough so that every component of $f^{-k}(U(w))$ that intersects $K$ is completely contained in $W$.

The sets $U(z)$ form an open covering of $f^{k}(K)$; let $\delta$ be its Lebesgue covering number. Now let $z_{0} \in K$ and $w_{0}:=f^{k}\left(z_{0}\right)$, and let $V$ be the component of $f^{-k}\left(\mathbb{D}_{\delta}\left(w_{0}\right)\right)$ containing $z$. Then $V \subset W$, so there is a branch $\varphi: \mathbb{D}_{\delta}\left(w_{0}\right) \rightarrow V$ of $f^{-k}$. Since $\left|\varphi^{\prime}(w)\right|<1 / \eta$ for all $w \in \mathbb{D}_{\delta}\left(w_{0}\right)$, it follows that $V \subset \mathbb{D}_{\delta}\left(z_{0}\right)$.

It now follows by induction that $\mathbb{D}_{\delta}\left(w_{0}\right)$ can be pulled back univalently along any backward orbit of $w_{0}$ that is contained in $K$. Hence $K \subset J_{r}(f)$, as claimed.

For the converse direction, let $K \subset J_{r}(f)$ be compact and forward-invariant. We may assume that $\vartheta:=\min _{z \in K}\left|f^{\prime}(z)\right| \leq 1$, as otherwise there is nothing to prove.

Let $z_{0} \in K$. It follows from Lemma 3.2 that $\lim \sup \left|\left(f^{n}\right)^{\prime}\left(z_{0}\right)\right|=\infty$, so we can pick some $n\left(z_{0}\right)$ and some open neighborhood $U\left(z_{0}\right)$ of $z_{0}$ such that $\left|\left(f^{n\left(z_{0}\right)}\right)^{\prime}(z)\right| \geq 2$ for all $z \in U\left(z_{0}\right)$.

Since $K$ is compact, it is covered by finitely many such neighborhoods; let us call them $U_{i}$ and denote the corresponding numbers $n\left(z_{0}\right)$ by $n_{i}$. Define $N:=\max _{i} n_{i}$, and let $m$ be sufficiently large that

$$
2^{m}>\frac{2}{\vartheta^{N}}
$$

Now we set $n:=m \cdot N$; we claim that $\left|\left(f^{n}\right)^{\prime}(z)\right|>2$ for all $z \in K$.

To prove this claim, define sequences $j_{k}$ and $z_{k}$ inductively by setting $z_{0}:=z$, choosing $j_{k}$ such that $z_{k} \in U_{j_{k}}$, and defining $z_{k+1}:=f^{n_{j_{k}}}\left(z_{k}\right)$.

Let $k$ be maximal with $p:=n-n_{j_{0}}-n_{j_{1}}-\cdots-n_{j_{k}} \geq 0$. Then $p<N$ and $k \geq m$ by choice of $n$. We can now write

$$
f^{n}(z)=f^{p}\left(z_{k}\right)=f^{p}\left(f^{n_{j_{k}}}\left(z_{k-1}\right)\right)=\cdots=f^{p}\left(f^{n_{j_{k}}}\left(f^{n_{j_{k-1}}}\left(\ldots\left(f^{n_{j_{0}}}(z)\right) \ldots\right)\right)\right) .
$$

So by the chain rule,

$$
\left|\left(f^{n}\right)^{\prime}(z)\right| \geq \vartheta^{p} \cdot 2^{k} \geq \vartheta^{N} \cdot 2^{m}>2
$$

by choice of $m$.

Proof of Theorem 1.2. We recall the setting of the theorem: $f$ is a nonconstant, nonlinear meromorphic function with $\mathcal{P}(f) \cap \mathbb{C}$ bounded, $K \subset \mathbb{C}$ is compact and forward-invariant and contains no critical points or parabolic periodic points. Also, 
no point of $K$ is the accumulation point of a recurrent critical orbit, or of a singular orbit contained in wandering domains.

We note that, since $\mathcal{P}(f)$ is bounded, the set of recurrent critical points is finite, and a singular value can be recurrent only if it is the image of a recurrent critical point. Furthermore, every nonrecurrent singular value is also strongly nonrecurrent. Hence every $z_{0} \in K$ satisfies the assumptions of Theorem 2.7, and therefore is regular in the sense of Definition 2.1. Let $\varepsilon>0$ be the Euclidean distance between $K$ and the set of critical points of $f$; by Lemma 2.8, there is a disk $U$ around $z_{0}$ such that every component of $f^{-n}(U)$ that intersects $K$ is mapped properly by $f^{n}$ and does not pass through any critical points. Hence every such pullback is univalent, and any point $z \in K$ that enters the disk $U$ infinitely many times belongs to $J_{r}(f)$.

Since every orbit in $K$ will accumulate on some $z_{0} \in K$, we see that $K \subset J_{r}(f)$. The claim that $K$ is hyperbolic now follows from the preceding lemma.

Branched versions of $J_{r}(f)$. In view of Mañé's theorem, we might want to consider analogs of the definition of $J_{r}(f)$ that do not require univalent pullbacks, but allow a bounded degree of branching. (Compare also [MM], where this is the definition used for the conical Julia set.)

3.5. Definition ( $\Delta$-branched radial Julia set). Let $\Delta \in \mathbb{N}$. We denote by $J_{r}^{\Delta}(f)$ the set of points $z \in J(f)$ with the following property. There is a number $\delta>0$ such that, for infinitely many $n$, the component of $f^{-n}\left(\mathbb{D}_{\delta}^{\#}\left(f^{n}(z)\right)\right)$ containing $z$ is simply connected and mapped by $f^{n}$ as a proper map of degree at most $\Delta$.

Note that, if $z_{0}$ is a regular point in the sense of Definition 2.1 and the orbit of $z$ is bounded and accumulates on $z_{0}$, then, by Lemma 2.8 $z \in J_{r}^{\Delta}(f)$ for some $\Delta \in \mathbb{N}$.

At least in the cases of interest to us, the extra generality does not gain us much in the set $J_{r}^{\Delta}(f)$.

3.6. Lemma $\left(J_{r}^{\Delta}(f)\right.$ and $\left.J_{r}(f)\right)$. Suppose that $J_{r}^{\Delta}$ has positive measure for some $\Delta \in \mathbb{N}$. Then $J(f)=\mathbb{C}$ and almost every point in $\mathbb{C}$ has a dense orbit.

In particular, if $\mathcal{P}(f) \neq \hat{\mathbb{C}}$, then $J_{r}^{\Delta}(f) \backslash J_{r}(f)$ has zero measure.

Proof. We can carry through the proof of Theorem 3.3 analogously (replacing Lemma 3.2 by a suitable version for branched pullbacks). It follows that almost every point in $\mathbb{C}$ has a dense orbit.

If $\mathcal{P}(f) \neq \hat{\mathbb{C}}$, then almost every orbit enters some disk in $\hat{\mathbb{C}} \backslash \mathcal{P}(f)$ infinitely many times, and this disk can be pulled back univalently as in the definition of $J_{r}(f)$. This proves the claim.

Remark. The condition $\mathcal{P}(f) \neq \hat{\mathbb{C}}$ is likely not necessary, but we only require the result in this case.

\section{LINE FIELDS}

A line field $\nu$ on a set $A$ is a measurable choice of a tangent line in each point of $A$. Equivalently, a line field is a measurable Beltrami differential $\mu d \bar{z} / d z$ with $|\mu|=1$. (See McM2, Section 3.5].) Such a line field $\mu$ is invariant under $f$ if $f^{*}(\mu)=\mu$ almost everywhere. We say that $f$ supports an invariant line field on the set $X$ if there is an invariant line field on some positive measure subset $A$ of $X$. 
4.1. Definition (Univalent line field). The line field $\mu$ is called univalent if for each point $z \in A$ there is a neighbourhood $U$ such that $\left.\mu\right|_{U}=\varphi^{*}(d \bar{z} / d z)$ for some univalent function $\varphi: U \rightarrow \mathbb{C}$. (Such a function $\varphi$ will be called a linearizing coordinate for $\mu$.)

Remark. Univalence of a line field is a local property; in particular, we do not require that the function $\varphi$ is defined on a neighborhood of $A$.

If the standard line field $d \bar{z} / d z$ is invariant under some analytic function $f$, then $f^{\prime}(z)$ must be a real constant, and hence $f$ is affine. It follows that the preimages of straight horizontal and vertical lines under linearizing coordinates $\varphi$ form two (transverse) analytic foliations on any set where $\mu$ is univalent. If $\mu$ is invariant under an analytic function $f$, then these foliations are also invariant.

Let us establish some preliminaries regarding line fields that are univalent everywhere; in particular we will show that an entire function cannot have an invariant line field that is univalent at every point of $\mathbb{C}$. Both of the following statements are implicit in GKŚ, Proof of Theorem 2], but we will state and prove them here explicitly for the reader's convenience.

4.2. Lemma (Univalent line fields on simply connected surfaces). Let $X$ be a simply connected Riemann surface (i.e., $X$ is either the plane, the sphere or the disk). Suppose that $\mu$ is a line field that is locally univalent on all of $X$. Then $\mu$ is the pullback of the standard line field $d \bar{z} / d z$ under a nonconstant analytic function $g: X \rightarrow \mathbb{C}$ with no critical points. In particular, $X$ cannot be the Riemann sphere.

Proof. Let $U_{1}, U_{2} \subset X$ be simply connected. Assume that $U:=U_{1} \cap U_{2}$ is connected, and that there are functions $\varphi_{j}: U_{j} \rightarrow \mathbb{C}$ satisfying

$$
\mu=\varphi_{j}^{*}(d \bar{z} / d z)
$$

We claim that $\varphi_{1}$ extends holomorphically to $U_{1} \cup U_{2}$, with the extension still satisfying (4.1).

Indeed, consider the map $\psi:=\varphi_{1} \circ \varphi_{2}^{-1}$, which maps $\varphi_{2}(U)$ to $\varphi_{1}(U)$ conformally. Then $\psi^{*}(d \bar{z} / d z)=d \bar{z} / d z$. This means that $\psi^{\prime}$ is a real constant on $\varphi_{2}\left(U_{1} \cap U_{2}\right)$ and therefore wherever $\psi$ is defined it is equal to some affine map $A$ of the form $A(z)=\lambda z+c($ with $c \in \mathbb{C}$ and $\lambda \in \mathbb{R})$. It follows that the map

$$
\varphi(z):= \begin{cases}\varphi_{1}(z) & z \in U_{1}, \\ A\left(\varphi_{2}(z)\right) & z \in U_{2}\end{cases}
$$

is the desired holomorphic extension of $\varphi_{1}$.

It now follows that $\varphi_{1}$ can be extended analytically along any path $\gamma \subset X$, with the extension satisfying (4.1). (We cover $\gamma$ by neighborhoods $U_{1}, U_{2}, \ldots, U_{n}$, with associated maps $\varphi_{j}$, such that $U_{j} \cap U_{j+1}$ is connected. Then we apply our observation above.) By the monodromy theorem, this extension is defined on all of $X$, and we are done.

4.3. Corollary (Entire functions with invariant univalent line fields). Let $f: \mathbb{C} \rightarrow$ $\mathbb{C}$ be a nonconstant entire function, and suppose there is a univalent line field $\mu$ on $\mathbb{C}$ that is invariant under $f$.

Then $f$ is an affine map.

Proof. By the previous lemma, $\mu=g^{*}(d \bar{z} / d z)$ for some nonconstant entire function $g$ with no critical points. We may suppose that $f$ has a repelling periodic point 
$z_{0}$ with $g^{\prime}\left(z_{0}\right) \neq 0$. (Otherwise, it follows directly that $f$ is affine.) By changing coordinates and passing to an iterate, we may suppose that $z_{0}=0, f(0)=0$ and $g(0)=0$. Let $\varphi$ be the branch of $g^{-1}$ with $\varphi(0)=0$. Then the locally defined function

$$
A:=g \circ f \circ \varphi
$$

preserves the standard line field, and hence is a global affine map:

$$
A(z)=\lambda z, \quad|\lambda|=\left|f^{\prime}(0)\right|>1, \quad \lambda \in \mathbb{R} .
$$

By the identity theorem, $g \circ f=A \circ g$ on all of $\mathbb{C}$, so $f$ and $A$ are semiconjugate.

If $z_{1}$ is another periodic point of $f$, say of period $n$, then $A^{n}\left(g\left(z_{1}\right)\right)=g\left(z_{1}\right)$, so $g\left(z_{1}\right)=0$. Hence the set of periodic points of $f$ is contained in the discrete set $g^{-1}(0)$, and therefore $f$ is affine.

Finally, we require some facts about pushforwards and pullbacks of univalent line fields by maps with a critical point. We begin with the following simple observation.

4.4. Observation (Critical pullbacks). Let $z, w \in \hat{\mathbb{C}}$, and let $f$ be holomorphic near $z$, with $f(z)=w$. Let $\nu$ be a line field near $w$, and let $\mu$ be the pullback of $\nu$ under $f$.

Suppose that $\mu$ is univalent near $z$. Then either $f^{\prime}(z) \neq 0$, and $\nu$ is univalent near $w$, or $z$ is a simple critical point of $f$, and $\nu$ is univalent on a punctured neighborhood of $w$, but not in $w$ itself.

Remark. In the case where $f$ is not univalent, so that $\nu$ is the pushforward of a univalent line field under a double cover, we say that $\nu$ has a one-pronged singularity near $w$.

Proof. By changing coordinates, we may assume that $z_{0}=w_{0}=0, \mu=d \bar{z} / d z$, and $f(z)=z^{d}$ for some $d \geq 1$. The line field $d \bar{z} / d z$ must then be invariant under $z \mapsto e^{2 \pi i / d} z$, which is only possible if $d \in\{1,2\}$, as required.

We will also be using the following well-known fact:

4.5. Lemma. There is no line field on the Riemann sphere that is univalent everywhere except (possibly) at a single one-pronged singularity.

Proof. By Lemma 4.2, there is no globally univalent line field on the sphere. So assume $\mu$ is a line field with a single one-pronged singularity. Without loss of generality, we may assume that this singularity is at $\infty$. Then the line field is univalent on $\mathbb{C}$ and has as its linearizing coordinate a global entire function $\varphi$ : $\mathbb{C} \rightarrow \mathbb{C}$. However, this is impossible, since there cannot be a linearizing coordinate defined on a complete punctured neighborhood of a one-pronged singularity (i.e., it is impossible to define the square root continuously on a punctured neighborhood of zero).

\section{Exceptional VAlues}

The big Picard theorem states that a holomorphic function cannot omit more than two values in the neighborhood of an essential singularity, and Montel's theorem states that any family of functions that omit the same three values is normal. We will require the following well-known fact from Nevanlinna theory (compare e.g. [B2]), which generalizes both theorems. 
5.1. Theorem (Branched Values). Let $a_{1}, \ldots, a_{r} \in \hat{\mathbb{C}}$ be distinct points, and let $\nu_{1}, \ldots, \nu_{r} \geq 1$ be integers such that

$$
\sum_{i=1}^{r}\left(1-\frac{1}{\nu_{i}}\right)>2
$$

(a) Let $f: \mathbb{C} \rightarrow \hat{\mathbb{C}}$ be a meromorphic function such that each $a_{i}$ has only finitely many preimages of multiplicity less than $\nu_{i}$. Then $f$ is rational.

(b) Let $U \subset \mathbb{C}$ be a domain. The family of all meromorphic functions $f: U \rightarrow \hat{\mathbb{C}}$ for which each preimage of each $a_{i}$ has multiplicity at least $\nu_{i}$ is a normal family in the sense of Montel.

Recall that the backward orbit of $z \in \hat{\mathbb{C}}$ under $f$ is the set

$$
O^{-}(z)=\left\{w \in \mathbb{C}: f^{n}(w)=z \text { for some } n \geq 0\right\}
$$

of iterated preimages of $z$ under $f$. If $w_{0} \in O^{-}(z)$, then the multiplicity of $w_{0}$ as an iterated preimage of $z$ is its multiplicity as a zero of the function $w \mapsto f^{n}(w)-z$ (where $n$ is chosen as small as possible). If the multiplicity of $w_{0}$ is 1 , we say that the preimage $w_{0}$ is unbranched; otherwise, $w_{0}$ is branched.

A value $z \in \hat{\mathbb{C}}$ is Fatou exceptional if its backward orbit is a finite set. We denote the set of all Fatou exceptional values by $E_{F}(f)$. Let us also introduce a related concept: we say that $z$ is branch exceptional if $z$ has only finitely many unbranched iterated preimages. We denote the set of all such values by $E_{B}(f)$. (This concept seems to appear implicitly in Schwick's simplified proof of the density of repelling periodic cycles in the Julia set $\mathrm{Sch}$. Also, it is somewhat related to that of univalently omitted values introduced in GKŚ.)

If $z \in E_{B}(f)$, let us define the exceptional multiplicity of $z$ as the largest number $2 \leq \nu \leq \infty$ such that $O^{-}(z)$ contains at most finitely many points of multiplicity less than $\nu$. (In particular, the Fatou exceptional values are exactly the branched exceptional values of exceptional multiplicity $\infty$.)

The following lemma is well known for Fatou exceptional points [M1, Lemma 4.9 and Theorem 4.10], but the corresponding statement about branch exceptional points does not seem to appear explicitly in the literature.

5.2. Lemma (Unbranched preimages of nonexceptional points are dense). Let $f: \mathbb{C} \rightarrow \hat{\mathbb{C}}$ be a nonconstant, nonlinear meromorphic function. For every branch exceptional value $v \in E_{B}(f)$, let $2 \leq \nu(v) \leq \infty$ be its exceptional multiplicity. Then

$$
\sum_{v \in E_{B}(f)}\left(1-\frac{1}{\nu(v)}\right) \leq 2
$$

In particular, $\# E_{B}(f) \leq 4$.

Furthermore, let $v \in \hat{\mathbb{C}}$, and let $z \in J(f)$. If $v \notin E_{F}(f)$, then every neighborhood $U$ of $z$ contains a point $w \in O^{-}(v)$. If additionally $v \notin E_{B}(f)$, then $w$ can be chosen to be unbranched.

Remark. The first part of the theorem can also be inferred directly from Theorem 5.1 for transcendental functions, while for rational functions it follows from elementary combinatorial considerations (see e.g. [KLR, Lemma 2.3]). Instead, we provide a simple unified proof of both statements, using Theorem 5.1 in all cases.

Proof. The theorem will be deduced from the following claim. 
Claim. Let $z \in J(f)$, and let $U$ be a neighborhood of $z$. Let $a_{1}, \ldots, a_{r} \in \hat{\mathbb{C}}$ be distinct points and suppose that there are $\nu_{1}, \ldots, \nu_{r} \in\{2,3, \ldots, \infty\}$ such that $O^{-}\left(a_{i}\right) \cap U$ contains no point of multiplicity less than $\nu_{i}$. Then $\sum_{i=1}^{r}\left(1-1 / \nu_{i}\right) \leq 2$. In particular, $r \leq 4$.

Proof. Let us first suppose that all iterates $f^{n}$ can be defined as meromorphic functions on $U$ (this is always the case if $f$ is rational or entire). Since $z \in J(f)$, these iterates do not form a normal family, and the claim follows directly from Theorem 5.1 (b).

Otherwise, $f$ is a transcendental meromorphic function, and $U$ contains a point $z_{1}$ with $f^{n}\left(z_{1}\right)=\infty$ for some $n \geq 0$. We can pick a small neighborhood $V \subset U$ of $z_{1}$ that contains no critical points or poles of $f^{n}$ apart from $z_{1}$. Let $W:=f^{n}(V)$; then $W$ is a neighborhood of $\infty$. By assumption, every point in $f^{-1}\left(a_{i}\right) \cap W$ has multiplicity at least $\nu_{i}$. Hence, the claim now follows from Theorem 5.1 (aia).

To deduce the first part of the theorem, let $a_{1}, \ldots, a_{r} \in E_{B}(f)$. Since the Julia set is uncountable, we can choose some $z \in J(f)$ that is not one of the finitely many iterated preimages of $a_{i}$ of multiplicity less than $\nu\left(a_{i}\right)$. We can now pick a small neighborhood $U$ of $z$ and apply the Claim.

To prove the second part, suppose $z \in J(f)$ and $v \notin E_{F}(f)$ (resp. $v \notin E_{B}(f)$ ). We can apply the Claim to five distinct iterated preimages (resp. unbranched iterated preimages) $v_{1}, \ldots, v_{5}$ of $v$. It follows that at least one of these must have an unbranched iterated preimage in $U$, as desired.

\section{UNIVALENT LINE FIELDS}

With these preliminaries, we are ready to prove the main fact required for the proof of Theorem 1.3, compare [McM2, Lemma 3.16].

6.1. Theorem (Maps supporting a univalent line field). Let $f: \mathbb{C} \rightarrow \hat{\mathbb{C}}$ be a nonconstant, nonlinear meromorphic function, and suppose that there is an invariant line field $\nu$ on $\widehat{\mathbb{C}}$ that is univalent on an open set $U$ intersecting $J(f)$.

Then $f$ is conjugate to one of the following:

(a) $z \mapsto z^{k}, k \in \mathbb{Z},|k| \geq 2$,

(b) $z \mapsto T_{k}(z)$ or $z \mapsto-T_{k}(z)$, where $k \geq 2$ and $T_{k}$ is the $k$-th Chebyshev polynomial, or

(c) a Lattès map.

(In particular, $f$ is a rational function.)

Proof. Let $\widetilde{U}$ be the set of points at which the line field $\nu$ is univalent, and set $K:=\hat{\mathbb{C}} \backslash \widetilde{U}$. Any point that has an unbranched iterated preimage in $U$ must clearly belong to $\widetilde{U}$. So it follows from Lemma 5.2 that $K \subset E_{B}(f)$.

Let us divide $K$ into two sets, by letting $K_{B}$ consist of all points of $K$ that have some iterated preimage under $f$ that belongs to $\widetilde{U}$, and setting $K_{F}=K \backslash K_{B}$. Since $K$ is finite, we have $K_{F} \subset E_{F}(f)$. Now let $w \in K_{B}$ and let $z \in \widetilde{U}$ be such that $f^{n}(z)=w$ for some $n \geq 1$. By Observation 4.4, $f^{n}$ has a simple critical point at $z$ and $\nu$ has a one-pronged singularity near $w$. The same argument, applied to $f^{n+1}$, also shows that $f\left(K_{B} \backslash\{\infty\}\right) \subset K_{B}$.

We set $S:=\hat{\mathbb{C}} \backslash K_{F}$ and summarize what we know so far:

(a) The line field $\nu$ is univalent at every point of $S \backslash K_{B}$. 
(b) $f\left(K_{B} \backslash\{\infty\}\right) \subset K_{B}$.

(c) The line field $\nu$ has a one-pronged singularity in every point of $K_{B}$.

(d) $f: S \backslash\{\infty\} \rightarrow S$ is holomorphic and preserves the line field $\nu$. In particular, $f$ has only simple critical points in $S$, and these are exactly the points in $f^{-1}\left(K_{B}\right) \backslash K_{B}$.

Points in $K_{F}$ have exceptional multiplicity $\infty$, while points in $K_{B}$ have exceptional multiplicity 2. Hence, according to Lemma 5.2 , we only have the following possibilities:

(a) $S$ is the punctured plane, and $K_{B}$ is empty;

(b) $S$ is the plane, and $0 \leq \# K_{B} \leq 2$;

(c) $S$ is the sphere, and $2 \leq \# K_{B} \leq 4$.

(Recall that the cases $S=\hat{\mathbb{C}}$ and $\# K_{B}=0,1$ cannot occur by Lemma 4.5.)

This amounts to saying that $S$ is an affine Thurston orbifold with only simple branch points, and $f: S \backslash\{\infty\} \rightarrow S$ is an analytic map between orbifolds. (Compare [McM2, Appendix A] or [M1, Appendix E].) Because of the above classification, the orbifold Euler characteristic is nonnegative (where we use the terminology from [M1, Appendix E]), and therefore the universal covering $X$ is conformally $\mathbb{C}$ or $\hat{\mathbb{C}}$.

In other words, there is a "universal cover" $X \in\{\mathbb{C}, \hat{\mathbb{C}}\}$ and an analytic function $\pi: X \rightarrow S$ that is completely ramified of degree 2 over all points of $K_{B}$, and a covering elsewhere (compare [M1, Appendix E] for the complete list of these covering maps). Since $\nu$ lifts to a univalent line field $\tilde{\nu}$ on all of $X$, it follows from Lemma 4.2 that we must have $X=\mathbb{C}$. According to [M1, Appendix E] this leaves only four cases: $S$ is the punctured plane and $\pi$ is the exponential map; $S$ is the plane and $\pi$ is a cosine map; $S$ is the sphere and $\pi$ is a Weierstraß $\wp$-function; $S$ is the plane and $\pi$ is the identity. By Corollary 4.3 and the assumption that $f$ is not affine, the last case does not occur.

Claim. $f$ lifts to an affine function under $\pi$. That is, there is an affine function $A: \mathbb{C} \rightarrow \mathbb{C}$ such that $f \circ \pi=\pi \circ A$.

Proof. If $\infty \notin S$ (in particular, when $f$ is entire), then $f$ is a self-map of $S$ and hence can be lifted via the universal cover $\pi$ to a holomorphic function $\hat{f}: \mathbb{C} \rightarrow \mathbb{C}$ with $f \circ \pi=\pi \circ \hat{f}$. This lift $\hat{f}$ preserves the univalent line field $\tilde{\nu}$ on $\mathbb{C}$ and hence is affine by Corollary 4.3 .

So suppose that $\infty \in S$. The main problem is to show that $f$ has no asymptotic values in $S$. To do so, recall that a univalent line field gives rise to horizontal and vertical foliations (corresponding to straight horizontal and vertical lines under any linearizing coordinate of the line field $\mu$ ). A map that preserves the line field also preserves these foliations. Let us say that $a \in S$ is a leafwise asymptotic value of $f$ if there is a piece $\gamma:[0,1) \rightarrow S \backslash\{\infty\}$ of a horizontal or vertical leaf with $\gamma(t) \rightarrow \infty$ and $f(\gamma(t)) \rightarrow a$ as $t \rightarrow 1$. Since we assumed that $\infty \in S, f$ has at most four leafwise asymptotic values of $f$ (one for each direction in which a horizontal or vertical leaf can approach $\infty$ ).

We claim that any asymptotic value in $\widetilde{U}$ must be a leafwise asymptotic value. Indeed, let $a \in \widetilde{U}$ be arbitrary and suppose that $a$ is not a leafwise asymptotic value. Let $Q \subset \widetilde{U}$ be a neighborhood of $a$ that corresponds to a square under the linearizing coordinates of the linefield $\mu$ near $a$ and that contains no leafwise asymptotic values. (Recall that $\widetilde{U}$ also contains no critical values of $f$.) 
The definition of a leafwise asymptotic value ensures that any branch of $f^{-1}$ at a point in $Q$ can be continued analytically along any vertical or horizontal leaf of the foliation in $Q$. But any germ that can be continued analytically along every horizontal and vertical line in a square can be continued analytically to the entire square. Hence every branch of $f^{-1}$ defined at a point of $Q$ can be extended to the entire neighborhood $Q$. This means that $Q$ contains no singular values of $f$. In particular, $a$ is not a singular value of $f$, as claimed.

So $f$ has at most finitely many asymptotic values. Suppose that $a \in S$ was an asymptotic value, and let $W \subset S$ be a small simply connected neighborhood of $a$ that contains no other critical or asymptotic values of $f$. Then every component of $f^{-1}(W)$ is either mapped to $W$ by a proper map with at most one critical point (in which case this component is bounded), or to $W \backslash\{a\}$ by a universal covering. Since we assumed that $a$ is an asymptotic value, there is at least one component $V$ of the latter kind. However, if $\gamma$ is a curve in $W$ tending to $a$ along a (horizontal or vertical) leaf, then this curve has countably many preimages in $W$, all tending to $\infty$. Since each of these preimages must itself be contained in a leaf of the foliation, this is clearly incompatible with the structure of the line field near infinity.

So $f$ has no asymptotic values. Hence $f \circ \pi$ is a covering map branched exactly over $K_{B}$, with all branched points being of degree 2 . In other words, $f \circ \pi$ is a covering from its domain of definition to the orbifold $S$. Therefore the function $\pi: \mathbb{C} \rightarrow S$ lifts to a map $B: \mathbb{C} \rightarrow \mathbb{C}$ with $f \circ \pi \circ B=\pi$. This map $B$ must then preserve the univalent line field $\pi^{*}(\mu)$ on $\mathbb{C}$, and hence is affine. $A=B^{-1}$ is the desired map. (Note that, in particular, $f$ must be a rational map.)

To complete the proof of Theorem 6.1, it suffices to examine the three possible choices of $\pi$. If $\pi$ is an exponential map, then $f$ is a power map; if $\pi$ is a cosine map, then $f$ is a Chebyshev polynomial; if $\pi$ is a $\wp$-function, then $f$ is a Lattès map.

\section{Proofs of the main theorems}

Proof of Theorem 1.3. Let $z \in J_{r}(f)$ be a density point of the line field, and let $D$ be a disk of univalence as in Lemma 3.2, By [McM2, Theorem 5.16], the line field is univalent on $D$. (Roughly speaking, the pushforward of the line field on $D_{n}$ to $D$ will be very close to a univalent line field, and the claim follows from a compactness argument.)

The claim now follows from Theorem 6.1

For future reference, we also note the following restatement of Theorem 1.3

7.1. Corollary (No line fields on $J_{r}(f)$ ). Let $f: \mathbb{C} \rightarrow \hat{\mathbb{C}}$ be a nonconstant, nonlinear entire or meromorphic function, and suppose that $f$ is not a Lattès map.

Then $f$ supports no invariant line fields on its radial Julia set $J_{r}(f)$.

Proof. If $J_{r}(f)$ has measure zero, then $f$ supports no invariant line fields on $J_{r}(f)$ by definition. Otherwise, $f$ is measurably transitive, and the claim follows from Theorem 1.3 .

To begin the proof of Theorem 1.1 we note that, under fairly general hypotheses, the escaping set of a meromorphic function does not support invariant line fields. 
7.2. Theorem (Invariant line fields on escaping sets). Let $f: \mathbb{C} \rightarrow \hat{\mathbb{C}}$ be a transcendental entire or meromorphic function. Suppose that all poles of $f$ have degree at most $\Delta \in \mathbb{N}$, and that the set $S(f) \backslash\{\infty\}$ is bounded.

Then the escaping set

$$
I(f):=\left\{z \in \mathbb{C}: f^{n}(z) \rightarrow \infty\right\}
$$

supports no invariant line fields.

Proof. Let $K>0$ be sufficiently large so that $|s|<K$ for all $s \in S(f) \backslash\{\infty\}$ and set $D_{0}:=\{|z|>K\} \cup\{\infty\}$. If $U$ is a component of $f^{-1}\left(D_{0}\right)$, then either $U$ is a logarithmic tract, i.e. $f: U \rightarrow D_{0} \backslash\{\infty\}$ is a universal covering, or $U$ is bounded and $f: U \rightarrow D_{0}$ is a branched covering with at most one critical point (this critical point, if it exists, being a multiple pole). By assumption, the degree of the map $f: U \rightarrow D_{0}$ is bounded by $\Delta$.

It is proved in [R1] that there are no invariant line fields supported on the set of escaping points $z \in I(f)$ for which $f^{n}(z)$ is contained in a logarithmic tract for all sufficiently large $n$. We will denote this set by $I_{\ell}(f)$.

For $R>0$, let us denote by $I_{R}$ the set of escaping points $z \in I(f)$ that satisfy $\left|f^{j}(z)\right| \geq R$ for all $j \geq 0$. We claim that, for sufficiently large $R$,

$$
I_{R} \backslash I_{\ell}(f) \subset J_{r}^{\Delta}(f) .
$$

To prove this, let $D=\{|z|>2 K\} \cup\{\infty\} \subset D_{0}$ be a second disk around $\infty$, and let $C=C(\Delta, 1 / 2)$ be the constant from Lemma 2.2. In the following, we will use the hyperbolic metric dist $D_{0}^{*}$ in the multiply connected domain $D_{0}^{*}:=D_{0} \backslash\{\infty\}$. If $V \subset D_{0}^{*}$ is a simply connected domain, we denote by $\widetilde{\operatorname{diam}}_{D_{0}^{*}}(V)$ the diameter of the preimage components of $V$ in the universal cover of $D_{0}^{*}$. More precisely, let $\tilde{V}$ be a component of $\exp ^{-1}(V)$; then $\widetilde{\operatorname{diam}}_{D_{0}^{*}}(V)$ is the diameter of $\tilde{V}$ in the hyperbolic metric of the half plane $\{\operatorname{Re} z>\log K\}$.

Claim. Let $R>0$ be chosen large enough. Suppose that $|z| \geq R$ and $f(z) \in D$. Let $W$ be the component of $f^{-1}(D)$ containing $z$.

(a) Suppose that $W$ is bounded. Then $\widetilde{\operatorname{diam}}_{D_{0}^{*}}(W) \leq C$.

(b) Suppose that $W$ is unbounded, and let $V \ni f(z)$ be a simply connected domain with $\widetilde{\operatorname{diam}}_{D_{0}^{*}}(V) \leq C$. Then the component $V^{\prime}$ of $f^{-1}(V)$ containing $z$ also satisfies $\widetilde{\operatorname{diam}}_{D_{0}^{*}}\left(V^{\prime}\right) \leq C$.

Proof. If $R$ is sufficiently large, then every bounded component $W^{\prime}$ of $f^{-1}\left(D_{0}\right)$ that contains a point of modulus at least $R$ is contained in $D_{0}$. (This is because only finitely many components of $f^{-1}\left(D_{0}\right)$ can intersect the compact set $\mathbb{C} \backslash D_{0}$.)

So, if $W$ is as in (国), then the component $W^{\prime}$ of $f^{-1}\left(D_{0}\right)$ that contains $W$ satisfies $W^{\prime} \subset D_{0}$. By Lemma 2.2, the hyperbolic diameter of $W$ in $W^{\prime}$ is bounded by $C$, and the claim follows.

In the case of (B) , the map $f: W \rightarrow D$ is a universal covering. It follows from the standard estimate on the hyperbolic metric in a simply connected domain M1, Corollary A.8] that the derivative of $f$ in $z$, measured in the hyperbolic metric of $D_{0}$, tends to $\infty$ as $|z| \rightarrow \infty$ in $W$. (Compare, for example, [EL3, Lemma 1] and [R1, Formula (2.4)].) The claim follows.

Suppose in the following that $R$ is chosen sufficiently large according to the claim, and additionally that every point $z$ with $|z|>R$ has $\operatorname{dist}_{D_{0}^{*}}(z, \partial D)>C$. 
Now, to prove (7.1), let $z \in I_{R} \backslash I_{\ell}(f)$. Then there are infinitely many $n$ such that $f^{n}(z)$ is not contained in a logarithmic tract. Let $V_{n+1}:=D$, and let $V_{j}$, for $j=0, \ldots, n$, be inductively defined as the component of $f^{-1}\left(V_{j+1}\right)$ containing $f^{j}(z)$. By assumption, $V_{n}$ is bounded, and hence $\widetilde{\operatorname{diam}}_{D_{0}^{*}}\left(V_{n}\right) \leq C$ by (国). In particular, $V_{n} \subset D$.

Using the claim, we see inductively that $\widetilde{\operatorname{diam}}_{D_{0}^{*}}\left(V_{j}\right) \leq C$ for $j=n, n-1, \ldots, 0$. The map $f^{n}: V_{0} \rightarrow V_{n}$ is a conformal isomorphism, so $f^{n+1}: V_{0} \rightarrow V_{n+1}$ has degree at most $\Delta$.

In summary, there are infinitely many $n$ such that $f^{n+1}$ takes some simply connected neighborhood of $z$ to $D$ as a proper map of degree at most $\Delta$. By definition, this means that $z \in J_{r}^{\Delta}(f)$, as desired.

Since every escaping point $z \in I(f)$ will eventually map to a point in $I_{R}$, we see that every point of $z \in I(f) \backslash I_{\ell}(f)$ either belongs to $J_{r}^{\Delta}(f)$ or is on the backward orbit of a critical point.

It follows that $I(f) \backslash I_{\ell}(f)$ has zero Lebesgue measure. Indeed, otherwise the set $J_{r}^{\Delta}(f)$ would have positive measure, and by Lemma 3.6, $I(f)$ then has zero measure, a contradiction. This completes the proof.

Remark 1. If we do not assume that there is a bound on the degree of the poles of $f$, then it need no longer be true that $I(f) \backslash I_{\ell}(f)$ has zero Lebesgue measure. Indeed, Bergweiler and Kotus [BK] Theorem 1.4] construct a transcendental meromorphic function $f$ for which $S(f) \backslash\{\infty\}$ is bounded such that $\infty$ is not an asymptotic value of $f$ but $I(f)$ has positive measure.

Remark 2. We note that the proof of Theorem 7.2 can be significantly simplified if we assume the hypotheses of Theorem 1.1 (which is the setting in which we will apply it).

For future reference, we also note the following generalization of Theorem 7.2 .

7.3. Theorem (Invariant line fields on generalized escaping sets). Let $f: \mathbb{C} \rightarrow \hat{\mathbb{C}}$ be a transcendental entire or meromorphic function, and let $P$ be a finite set of pre-poles of $f$, together with $\infty$, such that $f(P \backslash\{\infty\}) \subset P$. For $p \in P$, let $k_{p} \geq 0$ be such that $f^{k_{p}}(p)=\infty$, and set $k:=\max _{p} k_{p}$.

Suppose that $S(f) \backslash P$ is compact. Suppose furthermore that the local degree of $f$ near any point of $f^{-1}(P)$ is uniformly bounded by $\Delta \in \mathbb{N}$.

Then the set

$$
I^{P}:=I^{P}(f):=\left\{z \in \mathbb{C}: \operatorname{dist}^{\#}\left(f^{n}(z), P\right) \rightarrow 0\right\}
$$

supports no invariant line fields.

Remark. For an example of a family where a suitable $I^{P}(f)$ has full measure but $I(f)$ has zero measure, see $\mathrm{KU} 2$.

Proof. The proof is analogous to that of the previous theorem: indeed, we may consider it as an application of that theorem to an appropriate renormalization of $f$.

More precisely, let $D_{0}$ be a spherical disk around $\infty$. For $p \in P \backslash\{\infty\}$, let $D_{p}$ be the component of $f^{-k_{p}}\left(D_{0}\right)$ containing $p$. If $D_{0}$ was chosen sufficiently small, then $f^{k_{p}}: D_{p} \rightarrow D_{0}$ is a proper map, unbranched except possibly over infinity, $D_{p} \backslash\{p\}$ contains no singular values of $f$, and $D_{p} \cap D_{0}=\emptyset$. 
Now let $G_{p}$ be the union of all components of $f^{-1}\left(D_{p}\right)$ that are contained in $D_{0}$, and $G:=\bigcup_{p} G_{p}$. We define $g: G \rightarrow D_{0}$ by

$$
\left.g\right|_{G_{p}}:=\left.f^{k_{p}+1}\right|_{G_{p}} .
$$

Then every component $U$ of $G$ is simply connected, and either $g: U \rightarrow D_{0}$ is a proper map, unbranched except possibly over $\infty$, or $g: U \rightarrow D_{0} \backslash\{\infty\}$ is a universal covering. Note that, in the former case, the degree of this proper map is uniformly bounded by $\Delta^{\prime \prime}:=\Delta \cdot \Delta^{\prime}$, where $\Delta^{\prime}$ is the maximal order of a pre-pole in $E$.

We can now apply the same argument as in the previous theorem to show that the escaping set $I(g)$, i.e., those orbits that tend to infinity under iteration, supports no invariant line fields. (Note that the results of [R1] do not require the function to be globally defined; see [R1, Corollary 4.3].)

An invariant line field for $f$ on a positive measure subset of $I^{P}$ would restrict to an invariant line field for $g$ on a positive measure subset of $I(g)$, so the theorem is proved.

We now prove Theorem 1.1. Let us restate it here with the slightly weaker hypotheses of prohibiting only "bad" wandering domains in the sense of Section 2 , (Recall the remark in the introduction following Theorem 1.1.)

7.4. Theorem (Absence of line fields for nonrecurrent maps). Let $f: \mathbb{C} \rightarrow \widehat{\mathbb{C}}$ be a nonlinear and nonconstant meromorphic function. If $f$ is nonrecurrent and has no pre-poles of arbitrarily high order or bad wandering domains, then the Julia set of $f$ supports no invariant line fields.

Proof. We know that there are no invariant line fields on $J_{r}(f)$ and $I(f)$ by Corollary 7.1 and Theorem 7.2. We also know that, for all $\Delta \in \mathbb{N}$, the set $J_{r}^{\Delta}(f) \backslash J_{r}(f)$ has zero measure by Lemma 3.6. Hence the theorem will be established once we prove the following claim, which shows that $I(f)$ and $J_{r}^{\Delta}(f)$ cover all of $J(f)$ except for a countable set.

Claim. If $z \in J(f)$ is not a pre-pole or eventually mapped to a parabolic periodic orbit, then either $z \in I(f)$ or $z \in J_{r}^{\Delta}(f)$ for some $\Delta \in \mathbb{N}$.

Proof. It suffices to consider the case where $\operatorname{dist}^{\#}\left(f^{n}(z), \mathcal{P}(f)\right) \rightarrow 0$ (otherwise, we have $z \in J_{r}(f)$ by definition).

First consider the case where the orbit of $z$ is unbounded, but $z \notin I(f)$. Set $R:=$ $\max _{z \in \mathcal{P}(f) \backslash\{\infty\}}|z|+1$. Choose a small disk $D \subset\{z \in \mathbb{C}:|z|>R\} \cup\{\infty\}$ around $\infty$ such that no unbounded component of $f^{-1}(D)$ contains points of modulus less than $R$.

By assumption, there is a sequence $\left(n_{k}\right)$ such that $f^{n_{k}+1}(z) \in D$ and $f^{n_{k}+1}(z) \rightarrow$ $\infty$, but $\left|f^{n_{k}}(z)\right|<R$ for all $k$. If $D_{k}$ is the component of $f^{-\left(n_{k}+1\right)}(D)$ containing $z$, then $D_{k}$ is bounded and $f^{n_{k}+1}: D_{k} \rightarrow D$ is a branched covering map, unbranched except possibly over $\infty$. Furthermore, since $f$ does not have pre-poles of arbitrarily high order, the degree of this map is uniformly bounded. So $z \in J_{R}^{\Delta}(f)$, for a suitable $\Delta$, as claimed.

Now suppose that the orbit of $z$ is bounded, and let $K$ denote the $\omega$-limit set of $z$; i.e. $K$ is the set of limit points of the sequence $\left(f^{n}(z)\right)$. Since $z$ does not map to a parabolic periodic point by assumption, it follows that $K$ contains some point $z_{0}$ that is not a parabolic periodic point. (To see this, note that no parabolic point can be an isolated point of the $\omega$-limit set of $z$, and that the set of all parabolic 
points is countable and hence contains no perfect set.) The point $z_{0}$ satisfies the assumptions of Theorem 2.7. Since the orbit of $z$ is bounded, it follows from Lemma 2.8 that $z \in J_{r}^{\Delta}$ for some $\Delta \in \mathbb{N}$.

\section{ACKNowledgments}

We thank Walter Bergweiler, Adam Epstein, Janina Kotus, Volker Mayer, Phil Rippon and Mariusz Urbański for interesting discussions.

\section{REFERENCES}

AL. Artur Avila and Mikhail Lyubich, Hausdorff dimension and conformal measures of Feigenbaum Julia sets, J. Amer. Math. Soc. 21 (2008), no. 2, 305-363, arXiv:math.DS/0408290. MR2373353 (2009i:37112)

BKL. I. N. Baker, J. Kotus, and Lü Yinian, Iterates of meromorphic functions. IV. Critically finite functions, Results Math. 22 (1992), no. 3-4, 651-656. MR.1189754 (94c:58166)

B1. Walter Bergweiler, Iteration of meromorphic functions, Bull. Amer. Math. Soc. (N.S.) 29 (1993), no. 2, 151-188, arXiv:math.DS/9310226. MR1216719 (94c:30033)

B2. $\quad$, Bloch's principle, Comput. Methods Funct. Theory 6 (2006), no. 1, 77-108. MR2241035 (2007f:30045)

BK. Walter Bergweiler and Janina Kotus, On the Hausdorff dimension of the escaping set of certain meromorphic functions, Preprint, 2009, arXiv:0901.3014.

BM. Walter Bergweiler and Shunsuke Morosawa, Semihyperbolic entire functions, Nonlinearity 15 (2002), no. 5, 1673-1684. MR1925433 (2003e:37056)

Bo. Heinrich Bock, Über das Iterationsverhalten meromorpher Funktionen auf der Juliamenge, Aachener Beiträge zur Mathematik 23, RWTH Aachen, 1998.

BH. David A. Brannan and Walter K. Hayman, Research problems in complex analysis, Bull. London Math. Soc. 21 (1989), no. 1, 1-35. MR967787 (89m:30001)

BC. Xavier Buff and Arnaud Chéritat, Ensembles de Julia quadratiques de mesure de Lebesgue strictement positive, C. R. Math. Acad. Sci. Paris 341 (2005), no. 11, 669674. MR2183346 (2006k:37133)

CJY. Lennart Carleson, Peter W. Jones, and Jean-Christophe Yoccoz, Julia and John, Bol. Soc. Brasil. Mat. (N.S.) 25 (1994), no. 1, 1-30. MR1274760 (95d:30040)

DMNU. M. Denker, R. D. Mauldin, Z. Nitecki, and M. Urbański, Conformal measures for rational functions revisited, Fund. Math. 157 (1998), no. 2-3, 161-173, Dedicated to the memory of Wiesław Szlenk. MR.1636885 (99j:58122)

EL1. Alexandre È. Eremenko and Mikhail Yu. Lyubich, Examples of entire functions with pathological dynamics, J. London Math. Soc. (2) 36 (1987), no. 3, 458-468. MR918638 (89e:30047)

EL2. Dynamics of analytic transformations, Leningrad Math. J. 1 (1990), no. 3, 563-634. MR1015124 (91b:58109)

EL3. - Dynamical properties of some classes of entire functions, Ann. Inst. Fourier (Grenoble) 42 (1992), no. 4, 989-1020. MR.1196102 (93k:30034)

GKŚ. Jacek Graczyk, Janina Kotus, and Grzegorz Świątek, Non-recurrent meromorphic functions, Fund. Math. 182 (2004), no. 3, 269-281. MR2098781 (2005f:37092)

KLR. Jeremy Kahn, Mikhail Lyubich, and Lasse Rempe, A note on hyperbolic leaves and wild laminations of rational functions, J. Difference Equ. Appl. 16 (2010), no. 5-6, 655-665.

Ke. John L. Kelley, General topology, D. Van Nostrand Company, Inc., Toronto-New YorkLondon, 1955. MR0070144 (16:1136c)

Ki. Masashi Kisaka, Some conditions for semi-hyperbolicity of entire functions and their applications, in Y. Imayoshi, Y. Komori, M. Nishio, K. Sakan (eds.), Complex Analysis and its Applications, OCAMI Studies vol. 2, 241-247, Osaka Municipal Universities Press, 2008.

KU1. Janina Kotus and Mariusz Urbański, Geometry and ergodic theory of non-recurrent elliptic functions, J. Anal. Math. 93 (2004), 35-102. MR2110325 (2005j:37065) 
KU2. Geometry and dynamics of some meromorphic functions, Math. Nachr. 279 (2006), no. 13-14, 1565-1584. MR2269255 (2007k:37061)

L. M. Yu. Lyubich, On typical behavior of the trajectories of a rational mapping of the sphere., Sov. Math., Dokl. 27 (1983), 22-25. MR0687919 (84f:30036)

Ma. Ricardo Mañé, On a theorem of Fatou, Bol. Soc. Brasil. Mat. (N.S.) 24 (1993), no. 1, 1-11. MR:1224298 (94g:58188)

MM. Gaven J. Martin and Volker Mayer, Rigidity in holomorphic and quasiregular dynamics, Trans. Amer. Math. Soc. 355 (2003), no. 11, 4349-4363. MR1990755 (2004i:37095)

MR. Volker Mayer and Lasse Rempe, Conical rigidity for meromorphic and Ahlfors islands maps, Manuscript.

McM1. Curtis T. McMullen, Area and Hausdorff dimension of Julia sets of entire functions, Trans. Amer. Math. Soc. 300 (1987), no. 1, 329-342. MR871679 (88a:30057)

McM2. Complex dynamics and renormalization, Annals of Mathematics Studies, vol. 135, Princeton University Press, Princeton, NJ, 1994. MR.1312365 (96b:58097)

McM3. - Hausdorff dimension and conformal dynamics. II. Geometrically finite rational maps, Comment. Math. Helv. 75 (2000), no. 4, 535-593. MR.1789177 (2001m:37089)

M1. John Milnor, Dynamics in one complex variable, third ed., Annals of Mathematics Studies, vol. 160, Princeton University Press, Princeton, NJ, 2006. MR 2193309 (2006g:37070)

M2. _ On Lattès maps, Dynamics on the Riemann sphere, Eur. Math. Soc., Zürich, 2006, pp. 9-43, arXiv:math.DS/0402147 MR2348953(2009h:37090)

O. Yûsuke Okuyama, Linearization problem on structurally finite entire functions, Kodai Math. J. 28 (2005), no. 2, 347-358. MR.2153922 (2006d:37089)

Po. Christian Pommerenke, Boundary behaviour of conformal maps, Grundlehren der Mathematischen Wissenschaften, vol. 299, Springer-Verlag, Berlin, 1992. MR1217706 (95b:30008)

Pr. $\quad$ Feliks Przytycki, Conical limit set and Poincaré exponent for iterations of rational functions, Trans. Amer. Math. Soc. 351 (1999), no. 5, 2081-2099. MR.1615954 (99h:58110)

R1. Lasse Rempe, Rigidity of escaping dynamics for transcendental entire functions, Acta Math. 203 (2009), no. 2, 235-267. MR.2570071

R2. Lasse Rempe, Hyperbolic dimension and radial Julia sets of transcendental functions, Proc. Amer. Math. Soc. 137 (2009), no. 4, 1411-1420. MR2465667

RvS. Lasse Rempe and Sebastian van Strien, Density of hyperbolicity for classes of real transcendental entire functions and circle maps, Preprint, 2010, arXiv:1005.4627.

Sch. Wilhelm Schwick, Repelling periodic points in the Julia set, Bull. London Math. Soc. 29 (1997), no. 3, 314-316. MR.1435565 (97m:30029)

ST. Mitsuhiro Shishikura and Tan Lei, An alternative proof of Mañé's theorem on nonexpanding Julia sets, The Mandelbrot set, theme and variations, London Math. Soc. Lecture Note Ser., vol. 274, Cambridge Univ. Press, Cambridge, 2000, pp. 265-279. MR:1765093 (2001b:37074)

St. Gwyneth M. Stallard, The Hausdorff dimension of Julia sets of hyperbolic meromorphic functions, Math. Proc. Cambridge Philos. Soc. 127 (1999), no. 2, 271-288. MR1705459 (2001e:37060)

UZ1. Mariusz Urbański and Anna Zdunik, The finer geometry and dynamics of the hyperbolic exponential family, Michigan Math. J. 51 (2003), no. 2, 227-250. MR1992945 (2004d:37068)

UZ2. Geometry and ergodic theory of non-hyperbolic exponential maps, Trans. Amer. Math. Soc. 359 (2007), no. 8, 3973-3997. MR2302520(2008j:37098)

UZ3. Instability of exponential Collet-Eckmann maps, Israel J. Math. 161 (2007), 347-371. MR2350166 (2008m:37084)

Department of Mathematical Sciences, University of Liverpool, Liverpool L69 7ZL, UNITED KINGDOM

E-mail address: 1.rempe@liverpool.ac.uk

Mathematics Institute, University of Warwick, Coventry CV4 7AL, United Kingdom

E-mail address: strien@maths.warwick.ac.uk 